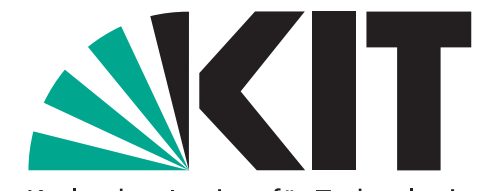

Karlsruher Institut für Technologie

\title{
The Condorcet set: majority voting over interconnected propositions
}

by Klaus Nehring, Marcus Pivato, Clemens Puppe

No. 51 | DECEMBER 2013

\section{WORKING PAPER SERIES IN ECONOMICS}

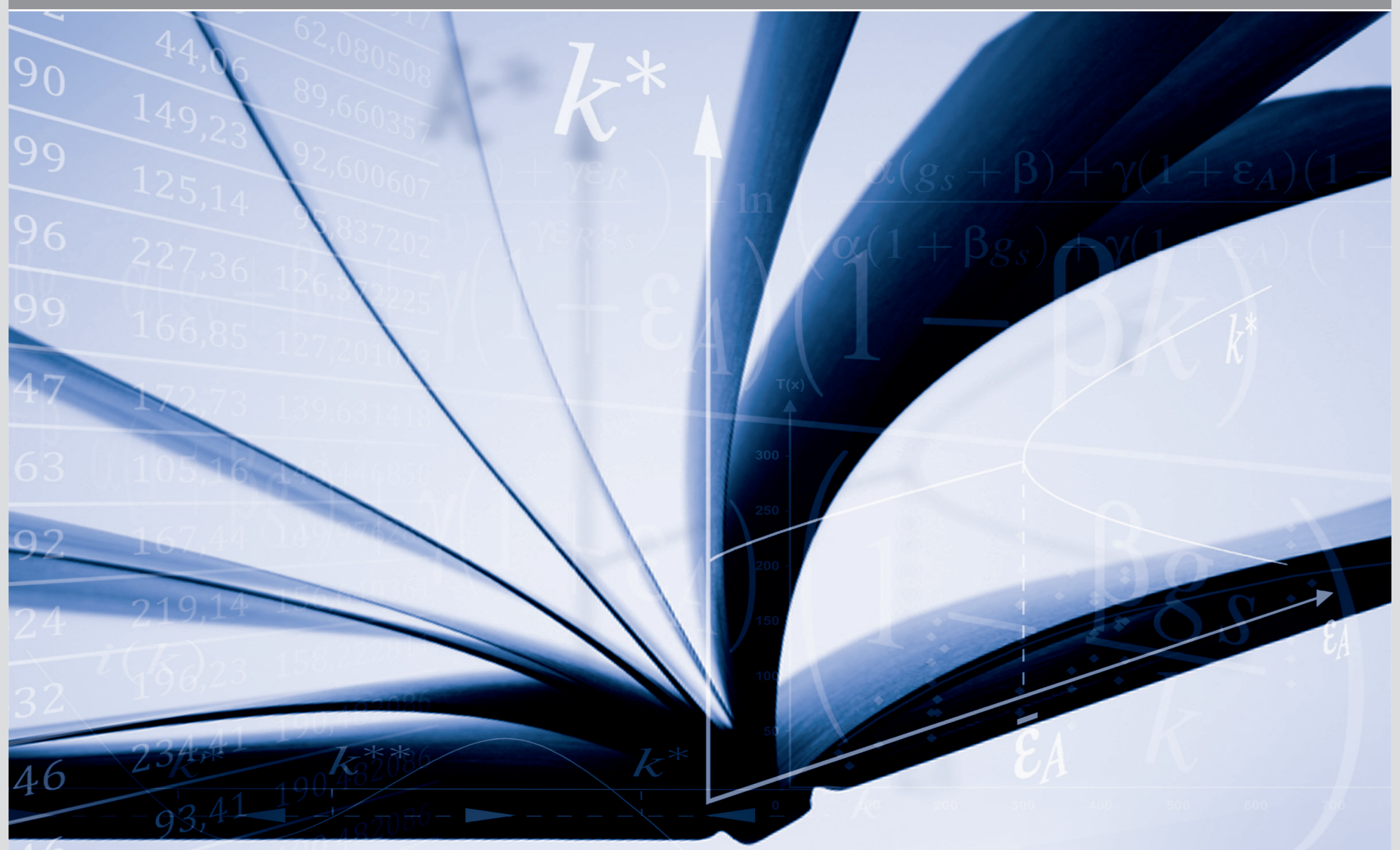




\section{Impressum}

Karlsruher Institut für Technologie (KIT)

Fakultät für Wirtschaftswissenschaften

Institut für Volkswirtschaftslehre (ECON)

Schlossbezirk 12

76131 Karlsruhe

KIT - Universität des Landes Baden-Württemberg und nationales Forschungszentrum in der Helmholtz-Gemeinschaft

Working Paper Series in Economics

No. 51, December 2013

ISSN 2190-9806

econpapers.wiwi.kit.edu 


\title{
The Condorcet set: Majority voting over interconnected propositions*
}

\author{
Klaus Nehring ${ }^{\dagger}$, Marcus Pivato ${ }^{\ddagger}$, and Clemens Puppe ${ }^{\S}$ \\ Revised version October 2013; first version: July 2011
}

\begin{abstract}
Judgement aggregation is a model of social choice in which the space of social alternatives is the set of consistent evaluations ('views') on a family of logically interconnected propositions, or yes/no-issues. Yet, simply complying with the majority opinion in each issue often yields a logically inconsistent collection of judgements. Thus, we consider the Condorcet set: the set of logically consistent views which agree with the majority on a maximal set of issues. The elements of this set are exactly those that can be obtained through sequential majority voting, according to which issues are sequentially decided by simple majority unless earlier choices logically force the opposite decision. We investigate the size and structure of the Condorcet set — and hence the properties of sequential majority voting — for several important classes of judgement aggregation problems. While the Condorcet set verifies McKelvey's (1979) celebrated 'chaos theorem' in a number of contexts, in others it is shown to be very regular and well-behaved.
\end{abstract}

In the context of preference aggregation, pairwise majority voting often fails to produce unambiguous outcomes because a Condorcet winner might not exist. Similarly, in the general judgement aggregation problem where each social outcome is described as a pattern

${ }^{*}$ We are grateful to an editor and a referee for their helpful comments and suggestions on an earlier version of this work. We would also like to thank audiences at the Workshop of the Centre of Mathematical Social Sciences at the University of Auckland, Tagung des Theoretischen Ausschusses des Vereins für Socialpolitik in Günzburg, the OECD conference "New Directions in Welfare" in Paris, the Meeting of the Society for Social Choice and Welfare in New Delhi, the COMSOC conference in Krakow, and of seminars at Université de Strasbourg, Université de Lausanne, Universität Wien, Ludwig-Maximilians-Univerisität München, Yale University, SUNY Stony Brook, Universität Graz, Universität Innsbruck and GRECAM in Marseille for comments and remarks.

${ }^{\dagger}$ Dept. of Economics, University of California at Davis, kdnehring@ucdavis.edu. Address: 1255 New Hampshire Ave NW, Apt. 825, Washington, DC 20036, U.S.A. Phone: (+1) 2022969360.

${ }^{\ddagger}$ Dept. of Mathematics, Trent University, Canada, marcuspivato@trentu.ca. Address: 1600 West Bank Drive, Peterborough, Ontario, Canada K9J 7B8. Phone: (+1) 7057481011.

${ }^{\S}$ Dept.of Economics and Business Engineering, Karlsruhe Institute of Technology, Germany, clemens.puppe@kit.edu. Address: Kaiserstrasse 12, 76128 Karlsruhe, Germany. Phone: (+49) 721 60843077 (corresponding author). 
of yes/no answers to certain interrelated propositions (a 'view'), issue-wise majority voting may fail to produce consistent collective 'views,' see Guilbaud (1952), Kornhauser and Sager (1986). Consistency is guaranteed only in those cases in which the aggregation problem has the structure of a median space, see Barthélémy and Monjardet (1981) and Nehring and Puppe (2007).

Does this mean that the very idea of majoritarian aggregation is ill-defined in the general case? Does 'inconsistency' imply 'impossibility'? While this is a possible reading of the Arrovian tradition in social theory, we submit that such a conclusion would be premature. While no view may be canonically privileged as 'uniquely majoritarian' in these cases, some views may still be 'more majoritarian' than others. We thus ask which views are 'most majoritarian.' As we shall see, this saves majoritarianism from impossibility, but at the cost of indeterminacy.

As a novel solution to the problem of inconsistency of issue-wise majority voting, we propose here the 'Condorcet set' and we argue that it serves as a natural demarcation criterion for majoritarian decisions. A collection of yes/no answers to all propositions is called a view; a view is consistent if it respects the logical interrelations between issues. A consistent view is more representative than another consistent view if its judgement on every issue is supported by at least as many voters, and on some issues by strictly more voters. A consistent view is Condorcet admissible if there is no other consistent view that is more representative. The set of such views is the Condorcet set. It is the set of all those views that can count as majoritarian. In particular, the Condorcet set always coincides with the issue-wise majority view whenever the latter is consistent.

The main goal of this paper is to investigate the properties of the Condorcet set, considered as a solution concept for judgement aggregation. A focal question concerns the size of the Condorcet set and thus the extent of majoritarian indeterminacy. We show that, frequently, the Condorcet set can be large. In particular, we focus on the question of when the Condorcet set at a given profile of individual views contains, for every proposition, both a view that affirms that particular proposition and a view that negates it. We refer to such profiles as globally indeterminate, and to aggregation problems as globally indeterminate if they admit globally indeterminate profiles. As our main result, we provide a general characterization of the set of indeterminate issues, with an attendant characterization of globally indeterminate profiles and globally indeterminate spaces. We then show that many interesting aggregation problems are indeed globally indeterminate, among them the preference aggregation problem, the aggregation of equivalence relations, resource allocation and many committee selections problems.

Majoritarian indeterminacy and, indeed, the full Condorcet set arises naturally from a 'diachronic' interpretation of the aggregation problem, in which decisions unfold over time and previous decisions potentially restrict later decisions due to the logical interrelation of propositions; a corresponding model of judgement aggregation has been studied by List (2004) and Dietrich and List (2007). Here, we show that a consistent view (over all issues) is an element of the Condorcet set if and only if there exists an ordering of issues (a 'path') such that the given view arises from 'sequential majority voting,' i.e. from deciding each issue by majority voting in the specified ordering provided that the issue is not yet determined by previous decisions. The size of the Condorcet set reflects the 
potential extent of path-dependence. In particular, an aggregation problem exhibits pathdependence at all whenever the Condorcet set has at least two elements, i.e. whenever (simultaneous) issue-by-issue majority voting is inconsistent.

How likely is global indeterminacy? Is it just a theoretical 'worst case,' or can it happen for plausible profiles? In the special case of preference aggregation, this question has been addressed in a rather dramatic fashion in the classical contribution of McKelvey (1979). Assuming a spatial structure of preferences on a topological space, McKelvey showed that, for generic profiles, majority voting is not just inconsistent, it is globally indeterminate. In this sense, global indeterminacy is almost bound to obtain.

With a finite set of alternatives, the dichotomy between consistency (hence complete determinacy) and global indeterminacy cannot be as clear-cut, even in the case of preference aggregation, as in McKelvey's continuous setting. We thus introduce a 'McKelvey property' that is applicable to the finite case and meant to capture the idea that global indeterminacies can arise from slight inconsistencies. Refining our main analysis of global indeterminacy, we show that some important aggregation problems have the McKelvey property, while others do not. The former include the preference aggregation and classification problems, the latter the committee selection and resource allocation problems. Thus, committee selection and resource allocation problems provide significant examples of spaces in which majority inconsistency is a problem, but a rather tame one. More broadly, our analysis shows that the qualitative behavior of majoritarian judgment aggregation strongly depends on the combinatorial structure of the space of feasible views.

\section{Related Literature}

The closest work to ours is the independent contribution by Lang et al. (2011) who introduced and analyzed a number of set-valued solution concepts based on the common denominator of 'minimization,' among them a solution concept equivalent to the Condorcet set. There is, however, no overlap in results between their work and ours. In particular, in contrast to their work, the present paper focuses on results supporting the Condorcet set as the natural demarcation criterion for 'majoritarian' aggregation, and on an analysis of indeterminacy, global indeterminacy, and path-dependence. Interestingly, even in the context of preference aggregation, the Condorcet set characterized in Proposition 3.1 of the present paper appears to be new in the literature.

The rest of the paper is organized as follows. In $\S 1$, we introduce terminology and define the Condorcet set. In $\S 2$ we consider (global) indeterminacy and state our main results. In $\S 3$, we explore indeterminacy and path-dependence in some paradigmatic judgement aggregation problems: preference aggregation, the aggregation of equivalence relations, committee selection and resource allocation. In the final $\S 4$ we consider the McKelvey

property and provide a classification of the examples in terms of this property. For ease of reading, all but the simplest proofs are relegated to an appendix at the end of the paper.

\section{1 'Majoritarian Views:' A Minimalist Definition}

In this section, we introduce our main new concept, the Condorcet set, and argue that it captures a natural criterion for majoritarianism. We start with a brief description of the 
framework of our analysis, the judgement aggregation model.

\subsection{The Judgment Aggregation Model}

A judgement aggregation problem is given by a set of $K$ propositions or issues. An element $\mathbf{x}=\left(x_{1}, x_{2}, \ldots, x_{K}\right) \in\{0,1\}^{K}$ is called an unconstrained view, or simply a view, and is interpreted as an assignment of a truth value of 'true' or 'false' to each proposition. ${ }^{1}$ Not all views are feasible, because there are logical constraints between the propositions (determined by the structure of the underlying decision problem faced by society). Let $X \subseteq\{0,1\}^{K}$ be the set of 'admissible' or consistent views; a given set $X \subseteq\{0,1\}^{K}$ is also referred to as an aggregation space. An anonymous profile is a probability measure on $X$ - that is, a function $\mu: X \longrightarrow[0,1]$ such that $\sum_{\mathbf{x} \in X} \mu(\mathbf{x})=1$ with the interpretation that, for all $\mathbf{x} \in X, \mu(\mathbf{x})$ is the proportion of the voters who hold the view $\mathbf{x}$. For any $Y \subseteq X$, define $\mu(Y):=\sum_{\mathbf{y} \in Y} \mu(\mathbf{y})$. Let $\Delta(X)$ be the set of all anonymous profiles. For any $\mu \in \Delta(X)$, any $k \in[1 \ldots K]$, let

$$
\mu_{k}(1):=\mu\left\{\mathbf{x} \in X ; x_{k}=1\right\}
$$

be the total 'popular support' for the position ' $x_{k}=1$,' and let $\mu_{k}(0):=1-\mu_{k}(1)$. Let $\Delta^{*}(X):=\left\{\mu \in \Delta(X) ; \mu_{k}(1) \neq \frac{1}{2}, \forall k \in[1 \ldots K]\right\}$ be the set of anonymous profiles where there is a strict majority supporting either 0 or 1 in each coordinate. ${ }^{2}$ An anonymous judgement aggregation rule is a correspondence (i.e. multivalued function) $F: \Delta^{*}(X) \rightrightarrows$ $\{0,1\}^{K}$. Most of the rules we will consider are single-valued, taking the form of a function $F: \Delta^{*}(X) \longrightarrow\{0,1\}^{K}$. For example, the issue-wise majoritarian judgement aggregation rule Maj : $\Delta^{*}(X) \longrightarrow\{0,1\}^{K}$ is defined as follows: for any $\mu \in \Delta^{*}(X), \operatorname{Maj}_{k}(\mu):=1$ if $\mu_{k}(1)>$ $\frac{1}{2}$, and $\operatorname{Maj}_{k}(\mu):=0$ if $\mu_{k}(1)<\frac{1}{2}$. For any $X$, denote by $\operatorname{Maj}(X):=\left\{\operatorname{Maj}(\mu) ; \mu \in \Delta^{*}(X)\right\}$ the set of all possible issue-wise majority views.

Say that a profile $\mu$ is majority determinate if $\operatorname{Maj}(\mu) \in X$, and say that an aggregation space $X$ is majority determinate if all profiles $\mu \in \Delta^{*}(X)$ are majority determinate. Unfortunately, it is quite common to find that $\operatorname{Maj}(\mu) \notin X$, i.e. there are in general profiles that are not majority determinate, because the 'majority ideal' can be inconsistent with the underlying logical constraints faced by society. ${ }^{3}$ What, in this case, should be considered to be the 'majority will'? This is the fundamental question to which we attempt to provide an answer in the subsequent analysis. Before we do so, let us consider for future reference a simple but fundamental special case, in which this problem does not arise.

\footnotetext{
${ }^{1}$ There are several different terms in the literature: e.g. Dokow and Holzman (2010) speak of 'binary evaluations,' List and Puppe (2009) use the term 'judgement set.'

${ }^{2}$ Usually, judgement aggregation is considered on all of $\Delta(X)$. However, a main goal in this paper is to investigate the multiplicity of solutions in the Condorcet set of a single profile; for this goal it is convenient to eliminate the 'spurious' multiplicities which arise when $\mu_{k}(1)=\frac{1}{2}$ for some $k \in[1 \ldots K]$. Thus, we will confine our attention to profiles in $\Delta^{*}(X)$ for expositional simplicity. If the set of voters is large (respectively odd), then a profile in $\Delta(X) \backslash \Delta^{*}(X)$ is highly unlikely (resp. impossible) anyways.

${ }^{3}$ Condorcet's 'voting paradox' is a well-known instance, see Section 3.1 below; another example that has received considerable interest recently is the so-called 'discursive dilemma,' see Kornhauser and Sager (1986), List and Pettit (2002), and List and Puppe (2009) for an overview of the subsequent literature.
} 
Example 1.1 (The Line) Say that $X$ is a linear aggregation problem, or simply a line, if $\mathbf{0}:=(0,0, \ldots, 0) \in X$ and the issues $[1 \ldots K]$ can be ordered in such a way that, for every view $\mathbf{x} \in X \backslash\{\boldsymbol{0}\}$ there exists $k_{\mathrm{x}} \in[1 \ldots K]$ such that $x_{j}=1$ for $j \leq k_{\mathbf{x}}$ and $x_{j}=0$ for $j>k_{\mathbf{x}}$. As is easily verified, $\operatorname{Maj}(\mu) \in X$ for all $\mu$ in this case, i.e. $X$ is majority determinate. Indeed, if one identifies $X$ with the set $\{0,1, \ldots, K\}$ by means of the mapping $\mathbf{x} \mapsto \#\left\{k \in[1 \ldots K]: x_{k}=1\right\}$, then $\operatorname{Maj}(\mu)$ is given by the median in the usual ordering of $[1 \ldots K]$ (i.e. the view $\mathbf{x} \in X$ such that $\mu(\{\mathbf{y} \in X: \mathbf{y} \geq \mathbf{x}\}) \geq \frac{1}{2}$ and $\left.\mu(\{\mathbf{y} \in X: \mathbf{y} \leq \mathbf{x}\}) \geq \frac{1}{2}\right){ }^{4}$ Concrete examples of this linear structure are views on the desirable inheritance tax rate, the maximal prison term served for a particular crime, the term limit for representative of government officials, or the number of days within which an abortion is legal.

Fig. 1 depicts a hypothetical case in which $20 \%$ of the population consider no inheritance tax as desirable, $35 \%$ consider an inheritance tax rate of 0.1 desirable, and $45 \%$ consider an inheritance tax rate of 0.5 desirable.

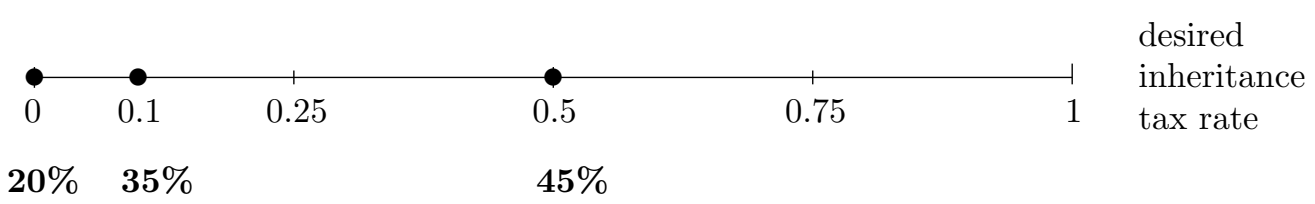

Figure 1: A linear aggregation problem

Evidently, the issue-wise majority view (i.e. the median) is at 0.1 (see Example 1.6 below for further discussion).

\subsection{Demarcating Majoritarianism}

How can we distinguish 'majoritarian' from 'non-majoritarian' aggregation rules? In this subsection we propose two basic principles that capture important aspects of majoritybased group decisions. Let us start with simplest cases first, and consider the following principle of 'plain' majoritarianism that one may impose on an aggregation rule $F$.

Plain Majoritarianism (PM) If $\mu(\mathbf{x})>\frac{1}{2}$ for some $\mathbf{x} \in X$, then $F(\mu)=\mathbf{x}$.

If $\# X=2$, this is of course the ordinary ('simple') majority criterion, shown by May (1952) to be the only neutral, anonymous and positively responsive aggregation method in this case. Plain Majoritarianism has specific further implications. In particular, in situations in which there are only two distinct views among the population, by PM, the group view must coincide with the view of the greater number. Thus PM does not see any need - or, more skeptically, does not leave any room - for compromise. While PM is thus a strong and specific requirement that might be questioned under some normative assumptions, we submit that it is a cogent expression of a basic intuition of a specifically

\footnotetext{
${ }^{4}$ Note that the median is unique due to our restriction to $\Delta^{*}(X)$.
} 
majoritarian outlook as classically expressed, for example, by the 17th century political philosopher Grotius according to whom "the majority would naturally have the right and authority of the whole," see (Risse, 2004, p.43). We thus take Plain Majoritarianism to be a fundamental minimal condition for demarcating whether or not a group choice is to be counted as majoritarian: by its very definition, a majority-based aggregation rule must satisfy PM. Whether or not majoritarianism itself is normatively compelling as a standard of group choice is a question that we do not address here. Indeed, our present goal is not to normatively justify majoritarian principles of judgment aggregation and social choice, but to determine the broad outlines of their content. For example, while the Borda rule is often viewed as a normatively attractive voting or preference aggregation rule, there is little question that it is not majoritarian in character. The justification of majoritarianism is, ultimately, a matter of political philosophy and not undertaken here. ${ }^{5}$

To illustrate the strength of PM, consider the following class of metric-based aggregation rules. Denote by $d_{H}$ the Hamming distance that takes the number of issues in which two views differ as their distance, formally $d_{H}(\mathbf{x}, \mathbf{y}):=\#\left\{k \in[1 \ldots K] ; x_{k} \neq y_{k}\right\}$ for all $\mathbf{x}, \mathbf{y} \in$ $\{0,1\}^{K}$. For $\beta>0$, consider the aggregation rules

$$
F_{\beta}(X, \mu):=\underset{\mathbf{x} \in X}{\operatorname{argmin}}\left(\sum_{\mathbf{y} \in X}\left[d_{H}(\mathbf{x}, \mathbf{y})\right]^{\beta} \cdot \mu(\mathbf{y})\right) .
$$

This class of aggregation rules contains interesting and important special cases. For example, as $\beta \rightarrow 0$, the rule $F_{\beta}$ converges to the plurality rule, which selects the view(s) that receive largest (relative) support. For $\beta=1$, the rule $F_{\beta}$ selects the consistent view(s) that minimize the average Hamming distance to the views of the voters. This is also known as the median rule and we will write Median $(X, \mu)$ for $F_{1}(X, \mu){ }^{6}$ If $X$ has the structure of a line as in Example 1.1, i.e. if $X$ is isomorphic to the set $\{1,2, \ldots, \# X\}$ viewed as a subset of the reals, then the Hamming distance coincides with the Euclidean distance and the median rule indeed selects the median in the usual sense.

For $\beta=2$, the aggregation rule $F_{\beta}$ selects the consistent view(s) that minimize the average of the squared Euclidean distance to the views of the voters. In case of a line, this amounts to the mean rule that selects (roughly) the average of the individual views. Finally, as $\beta \rightarrow \infty, F_{\beta}$ converges to the rule that minimizes the maximal 'disagreement' of any individual, selecting the midpoint(s) of the two most extreme views.

For any $\mathbf{x}, \mathbf{y}, \mathbf{z} \in\{0,1\}^{K}$, say that $\mathbf{y}$ is between $\mathbf{x}$ and $\mathbf{z}$ if, for all $k \in[1 \ldots K],\left(x_{k}=\right.$ $\left.z_{k}=0\right) \Longrightarrow\left(y_{k}=0\right)$ and $\left(x_{k}=z_{k}=1\right) \Longrightarrow\left(y_{k}=1\right)$. Thus, a view is between two other views if it coincides with them on all issues on which they agree. Furthermore, say that $\mathbf{y}$ is properly between $\mathbf{x}$ and $\mathbf{z}$ if, in addition, $\mathbf{x} \neq \mathbf{y} \neq \mathbf{z}$. For any $\mathbf{x} \in X$ and $\mathbf{z} \in\{0,1\}^{K}$,

\footnotetext{
${ }^{5}$ See Waldron (1999) for a prominent contemporary defense of majoritarianism, and Risse (2004) for a critique well-informed by social choice theory. Baldiga (2012) discusses problems of majoritarian principles in the context of direct vs. representative democracy.

${ }^{6}$ In the case of preference aggregation (see $\S 3.1$ below), the Hamming distance is also known as the Kemeny distance, and the median rule as the Kemeny rule (Kemeny, 1959), which has been axiomatically characterized by Young and Levenglick (1978). As a general-purpose judgement aggregation rule, the median rule has been studied by Barthélémy and Monjardet (1981, 1988), Barthélémy (1989) and Barthélémy and Janowitz (1991).
} 
write $\mathbf{x} \asymp \mathbf{z}$ if there exists no $\mathbf{y} \in X$ which is properly between $\mathbf{x}$ and $\mathbf{z}$. Finally, say that $X$ is regular if there are three views $\mathbf{x}, \mathbf{y}$ and $\mathbf{z}$ in $X$ such that $\mathbf{y}$ is properly between $\mathbf{x}$ and z.

Proposition 1.2 If $X$ is regular, then $F_{\beta}$ satisfies $P M$ if and only if $\beta \leq 1$

Note that condition Plain Majoritarianism does not use in any way the propositional structure of the underlying space $X$. But with this structure one can formulate stronger criteria for majority decisions. The following is particularly natural.

Condorcet Consistency (CC) If $\operatorname{Maj}(\mu) \in X$, then $F(\mu)=\operatorname{Maj}(\mu)$.

Clearly, condition CC implies PM but it can be decisive in many cases in which PM has no bite. If the underlying space is majority determinate, then condition $\mathrm{CC}$ is even decisive for all profiles. The additional force of CC over PM is evident from the following result which characterizes the metric-based aggregation rules that satisfy CC.

Proposition 1.3 If $X$ is regular, then $F_{\beta}$ satisfies $C C$ if and only if $\beta=1$

Thus, the median rule is in fact the only metric-based aggregation rule that satisfies CC. In particular, in regular spaces, plurality rule (i.e. $\lim _{\beta}{ }^{0} F_{\beta}$ ) does not satisfy condition $\mathrm{CC}$, and can on these grounds not be counted as a majoritarian aggregation rule. ${ }^{7}$

\subsection{Condorcet admissibility and the Condorcet Set}

Recall that $\mu_{k}(1)$ (resp. $\mu_{k}(0)$ ) denotes the popular support for position 1 (resp. 0) in issue $k$ at the profile $\mu$. Thus, for every view $\mathbf{x} \in X, \mu_{k}\left(x_{k}\right)$ is the popular support for the position specified by $\mathbf{x}$ in issue $k$. Say that view $\mathbf{x}$ is more representative of $\mu$ than view $\mathbf{y}$, denoted by $\mathbf{x} R(\mu) \mathbf{y}$, if it receives greater popular support in every issue. Formally,

$$
\mathbf{x} R(\mu) \mathbf{y} \quad: \Leftrightarrow \quad\left[\mu_{k}\left(x_{k}\right) \geq \mu_{k}\left(y_{k}\right) \text { for all } k \in K\right]
$$

We propose a conception of majoritarianism according to which more representative views are more majoritarian. In this paper, we explore the minimalist or broad-tent formulation of this conception which relies only on $R(\mu)$ as yardstick of comparative representativeness. Then the $R(\mu)$-maximal views can be considered to be the 'most majoritarian.' Henceforth, we will thus refer to the $R(\mu)$-maximal views as the Condorcet admissible views, and to their set as the Condorcet set, denoted by Cond $(X, \mu)$.

While the relation $R(\mu)$ is motivated in terms of comparative representativeness, we do not claim here that $R(\mu)$ represents the only possible formalization of ordinary intuitions and usages of 'representativeness.' Special - and crucial - to the relation $R(\mu)$ is the vector comparison reflecting a 'multi-criteria' conception of representativeness, the criteria being the alignment of a view with the distribution of individual judgements in each issue. As demonstrated by the following results, it is this multi-criteria structure which ensures that

\footnotetext{
${ }^{7}$ Plurality rule does satisfy condition $\mathrm{CC}$ in some non-regular spaces, for instance in the space $X=$ $\{(1,0,0),(0,1,0),(0,0,1)\}$, and more generally, in the spaces $X_{1,1 ; K}^{\text {com }}$ introduced in $\S 3.3$ below.
} 
'most representative' views can indeed count as majoritarian. In particular, by Lemma 1.5 below, Condorcet admissibility ensures Condorcet Consistency and, a fortiori, Plain Majoritarianism. ${ }^{8}$

Note that our definition of comparative representativeness allows for the full quantitative information entailed by a profile to be useable a priori. However, it turns out that the relation $R(\mu)$ is completely determined by the set of issues in which there is agreement with $\operatorname{Maj}(\mu)$. Specifically, we have the following simple result.

Lemma 1.4 Let $\mu \in \Delta^{*}(X)$. For $\mathbf{x} \in X$, let $\mathcal{M}(\mathbf{x}, \mu):=\left\{k \in[1 \ldots K] ; x_{k}=\operatorname{Maj}_{k}(\mu)\right\}$ be the set of issues in which $\mathbf{x}$ coincides with the 'majority ideal.' Then,

$$
\mathbf{x} R(\mu) \mathbf{y} \quad \Leftrightarrow \quad \mathcal{M}(\mathbf{x}, \mu) \supseteq \mathcal{M}(\mathbf{y}, \mu) .
$$

In particular, a view is Condorcet admissible if and only if it coincides with the 'majority ideal' on a maximal subset of issues.

Condorcet admissibility is proposed here as a necessary and sufficient condition for 'majoritarianism' in the sense that, for any given profile, a view can be counted as majoritarian if and only if it is an element of the Condorcet set. The necessity part of this claim follows almost by definition: a decision cannot plausibly be viewed as majority-based if it is possible to increase the popular support on an issue without decreasing it on any other issue. Equivalently, by Lemma 1.4, a decision cannot be counted as 'majoritarian' if it is possible to respect the majority view on a strictly larger set of issues. Moreover, Lemma 1.5 below characterizes the Condorcet admissible views as 'best approximations' to the majority ideal. ${ }^{9}$ The sufficiency of Condorcet admissibility as a criterion of majoritarianism is supported by the characterizations entailed by Propositions 1.7 and 1.8 below, either of which shows that every Condorcet admissible view has strong majoritarian credentials.

The following lemma yields a simple but fundamental characterization of the Condorcet set as the set of views that are 'best approximations' to the majority ideal; recall that $\mathbf{x} \asymp \mathbf{y}$ if no view is properly between $\mathbf{x}$ and $\mathbf{y}$ ( $\mathbf{x}$ is 'adjacent' to $\mathbf{y}$ ).

Lemma $1.5 \quad$ (a) If $\operatorname{Maj}(\mu) \in X$, then $\operatorname{Cond}(X, \mu)=\{\operatorname{Maj}(\mu)\}$.

(b) Otherwise, Cond $(X, \mu)=\{\mathbf{x} \in X$; $\mathbf{x} \asymp \operatorname{Maj}(\mu)\}$.

In this case, $\mid$ Cond $(X, \mu) \mid \geq 3$.

\footnotetext{
${ }^{8}$ One might consider, alternatively, employing a metric approach on which the representativeness of a view is measured by the average (transformed) distance from the individual views. As illustrated by the discussion of transforms of the Hamming distance above, unless the minimand is the untransformed average Hamming distance, the most representative views will not be Condorcet Consistent, cf. Proposition 1.3. Thus, whatever their merit, such approaches cannot serve as alternative routes to articulating majoritarianism in judgment aggregation.

${ }^{9}$ Lang et al. (2011) define their version of the Condorcet set in terms of the majority ideal. We think of this as less attractive, as it uses majoritarian intuitions on the right-hand side, and a priori rules out the use of quantitative information.
} 
Example 1.6 (The Median vs. the Mean in the Line) Consider again the line, identified with the set $\{0,1, \ldots, K\}$ as in Example 1.1, so that the view 0 corresponds to the rejection of all issues, and every view $k \geq 1$ corresponds to the affirmation of exactly the issues $\leq k$. Let $K \geq 3$, and suppose that under the profile $\mu$, the view 0 is supported by two agents, while each of the views $K-2, K-1$ and $K$ is supported by one agent, respectively. Then, in accordance with Lemma 1.5, $\operatorname{Maj}(\mu)=K-2$ (the 'median' view) is the single most representative view. Indeed, it receives a uniform 3/5-majority on each issue; any other feasible view looses against a majority on some issue, and no view reaches a larger majority on any issue. In particular, the median view $K-2$ is more representative than the 'mean view,' i.e. the view closest to the average of the individual views $(=(3 K-3) / 5)$ whenever $K \geq 5$. Note that the median view is also more representative than the "plurality view' 0 (the single view supported by the largest fraction of agents).

In some settings, the line is only defined qualitatively in terms of the implied betweenness relation among views, for example, if views are ordered in terms of some right to left spectrum of political values. In those cases, a mean may be formally defined but will hinge on an arbitrary cardinalization of distances. But even in settings in which the line represents a (discrete) quantity, there may exist other scales that could be viewed as equally or more appropriate as a basis for averaging. For instance, the value of the mean in Fig.1 above (at 0.26) depends on the chosen scale in terms of (absolute) levels of the tax rate. By contrast, the median (at 0.1) is invariant with respect to the choice of the scale. From the perspective of representing the 'people's will,' the median has thus greater appeal in this example than the mean; clearly, it also has much greater appeal than the 'plurality view' (at 0.5 ).

An aggregation rule $F: \Delta^{*}(X) \rightrightarrows\{0,1\}^{K}$ is called Condorcet admissible if $F(\mu) \subseteq$ Cond $(X, \mu)$ for any $X$ and $\mu$. A natural class of Condorcet admissible rules arises in the following way. Consider a gain function $\phi:\left[-\frac{1}{2}, \frac{1}{2}\right] \longrightarrow \mathbb{R}$, assumed to be non-decreasing and odd (i.e. $\phi(r)=-\phi(-r)$ ) with $\phi(r)<0$ for all $r<0$ and $\phi(r)>0$ for all $r>0$. For any such function, and strictly positive weights $\lambda_{k}>0$ with $\sum_{k \in[1 \ldots K]} \lambda_{k}=1$, define the weighted additive majority rule $F_{\phi, \lambda}: \Delta^{*}(X) \rightrightarrows X$ by

$$
F_{\phi, \lambda}(\mu):=\underset{\mathbf{x} \in X}{\operatorname{argmax}} \sum_{k \in[1 \ldots K]} \lambda_{k} \cdot \phi\left[\mu_{k}\left(x_{k}\right)-\frac{1}{2}\right] .
$$

In particular, if $\phi(r)=\operatorname{sign}(r)$ for all $r \in\left[-\frac{1}{2}, \frac{1}{2}\right]$ and $\lambda_{k}=1 / K$ for all $k$, then $F_{\phi, \lambda}$ is the Slater rule:

$$
\text { Slater }(X, \mu):=\underset{\mathbf{x} \in X}{\operatorname{argmax}} \#\left\{k \in[1 \ldots K] ; \mu_{k}\left(x_{k}\right)>\frac{1}{2}\right\} .
$$

The Slater rule selects the consistent views that maximize the number of issues in which there is agreement with the majority will. ${ }^{10}$ This rule was first suggested by Slater (1961) in the setting of Arrovian preference aggregation, in which it selects the transitive orderings that agree with the majority tournament in the largest number of binary comparisons.

\footnotetext{
${ }^{10}$ It is also easily verified that $\operatorname{Slater}(X, \mu)=\operatorname{argmin}_{\mathbf{x} \in X} d_{H}[\mathbf{x}, \operatorname{Maj}(\mu)]$; that is, the Slater rule selects the consistent view $(\mathrm{s})$ that minimize the Hamming distance to the issue-wise majority view.
} 
On the other hand, it is easily verified that if $\phi(r)=r$ for all $r \in\left[-\frac{1}{2}, \frac{1}{2}\right]$ and $\lambda_{k}=1 / K$ for all $k$ then the corresponding $F_{\phi, \lambda}$ coincides with the median rule defined above, i.e.

$$
\operatorname{Median}(X, \mu)=\underset{\mathbf{x} \in X}{\operatorname{argmax}} \sum_{k \in[1 \ldots K]} \mu_{k}\left(x_{k}\right) .
$$

This alternative representation of the median rule underlines its importance in the theory of aggregation. More generally, the following result establishes a close connection between the class of weighted additive majority rules and Condorcet admissibility.

Proposition 1.7 Any weighted additive majority rule $F_{\phi, \lambda}$ is Condorcet admissible. Conversely, for any $\mu \in \Delta^{*}(X)$, any $\mathbf{x} \in \operatorname{Cond}(X, \mu)$, and any gain function $\phi$, there exist weights $\lambda_{k}>0$ such that $F_{\phi, \lambda}(\mu)=\{\mathbf{x}\}$.

\subsection{A Diachronic Interpretation: Sequential Majority Voting}

Condorcet admissibility is closely related to 'sequential' majority voting, according to which issues are decided sequentially by simple majority unless previous decisions force the opposite choice. Specifically, we show now that a view $\mathbf{x}$ is Condorcet admissible if and only if there exists a sequence of issues ('a path') $\gamma$ such that $\mathbf{x}$ is the result of sequential majority voting along the path $\gamma$. From an applied viewpoint, this 'diachronic' perspective on the judgement aggregation problem is important since, in reality, many social decisions are made in a piecemeal fashion, with different aspects decided at different times.

For any $\mathbf{y}=\left(y_{k}\right)_{k=1}^{K} \in\{0,1\}^{K}$ and $J \subset[1 \ldots K]$, define $\mathbf{y}_{J}:=\left(y_{j}\right)_{j \in J} \in\{0,1\}^{J}$. For any $i \in[1 \ldots K] \backslash J$, say that $y_{i}$ is $X$-consistent with $\mathbf{y}_{J}$ if there exists some $\mathbf{x} \in X$ with $\mathbf{x}_{J}=\mathbf{y}_{J}$ and $x_{i}=y_{i}$-otherwise $y_{i}$ is $X$-inconsistent with $\mathbf{y}_{J}$. A path through $[1 \ldots K]$ is a bijection $\gamma:[1 \ldots K] \longrightarrow[1 \ldots K]$. We now define the $\gamma$-sequential majority rule $F^{\gamma}: \Delta^{*}(X) \longrightarrow X$. Let $\mu \in \Delta^{*}(X)$ and let $\mathbf{z}:=\operatorname{Maj}(\mu)$. Define $\mathbf{y}:=F^{\gamma}(\mu) \in X$ inductively as follows:

- Define $y_{\gamma(1)}:=z_{\gamma(1)}$.

- Inductively, let $J:=\{\gamma(1), \gamma(2), \ldots, \gamma(n)\}$, and suppose we have already decided $\mathbf{y}_{J}$. Let $i:=\gamma(n+1)$. If $z_{i}$ is $X$-consistent with $\mathbf{y}_{J}$, then set $y_{i}:=z_{i}$. Otherwise, set $y_{i}=\neg z_{i} \cdot 11$

A key issue in the context of sequential majority voting is path-dependence (List, 2004; Dietrich and List, 2007). Say that the profile $\mu$ is path-dependent if there exist paths $\gamma$ and $\xi$ such that $F^{\gamma}(\mu) \neq F^{\xi}(\mu)$. Our next result establishes that the Condorcet admissible views are exactly those that can be obtained as the result of sequential majority voting:

Proposition 1.8 Let $X \subseteq\{0,1\}^{K}$.

(a) For any path $\gamma$ through $[1 \ldots K]$, the rule $F^{\gamma}$ is Condorcet admissible.

(b) Conversely, for any $\mu \in \Delta^{*}(X)$ and $\mathbf{x} \in \operatorname{Cond}(X, \mu)$, there exists a path $\gamma$ such that $F^{\gamma}(\mu)=\mathbf{x}$.

\footnotetext{
11 “ $\neg$ " represents logical negation. That is: $\neg 1:=0$ and $\neg 0:=1$.
} 
This result can be viewed as a judgement aggregation counterpart to a well-known characterization of the top cycle as the outcome of non-strategic voting in simple binary tree agendas due to Miller (1977). Propositions 1.7 and 1.8 lend support to our argument for the sufficiency of Condorcet admissibility as a criterion of majoritarian decisions, since either result shows that every Condorcet admissible view is supportable by rules with unquestionable majoritarian credentials. ${ }^{12}$

There are two different interpretations of how the decision paths emerge in the diachronic model, the 'contingency' and the 'design' interpretation. Under the former, the path is exogenously given, or chosen by history; in this case, the 'size' of the Condorcet set, i.e. the extent of path-dependence, measures the role of historical accident. Under the latter, the path is chosen by an agenda setter and the Condorcet set can be viewed as her option set, given the constraint of using simple majority voting in each single step of the decision sequence. In this case, the size of the Condorcet set measures the extent to which the agenda setter can manipulate the final outcome.

Under either interpretation, an important task is provide quantitative measures of 'size' and the implied extent of path-dependence and indeterminacy. This is indeed the central aim of our analysis in Section 2 below. For comparison, we conclude our exposition of the general model with a characterization of the spaces in which there is no path-dependence, and thus also no indeterminacy.

A profile $\mu \in \Delta^{*}(X)$ is path-independent if $F^{\gamma}(\mu)=F^{\xi}(\mu)$ for any two paths $\gamma$ and $\xi$ through $[1 \ldots K]$. The space $X$ itself is called path-independent if every $\mu \in \Delta^{*}(X)$ is pathindependent. Let $J \subseteq[1 \ldots K]$ and consider $\mathbf{w} \in\{0,1\}^{J}$ which corresponds to a subset of judgements on the issues in $J$. The set $J$ is the support of $\mathbf{w}$, denoted $\operatorname{supp}(\mathbf{w})$. We define the order of $\mathbf{w}$ as $\# \mathbf{w}:=\# J$. If $I \subseteq J$ and $\mathbf{v} \in\{0,1\}^{I}$, then we say $\mathbf{v}$ is a fragment of $\mathbf{w}$ (and write $\mathbf{v} \sqsubseteq \mathbf{w}$ ) if $\mathbf{v}=\mathbf{w}_{I}$. Furthermore, $\mathbf{w}$ is a forbidden fragment for $X$ if, for all $\mathbf{x} \in X$, we have $\mathbf{x}_{J} \neq \mathbf{w}$. Finally, $\mathbf{w}$ is a critical fragment if it is a 'minimal' forbidden fragment — that is, $\mathbf{w}$ is forbidden, and there exists no proper subfragment $\mathbf{v} \sqsubset \mathbf{w}$ such that $\mathbf{v}$ is forbidden. Let $W(X)$ be the set of critical fragments for $X$ and let $\kappa(X):=\max \{\# \mathbf{w} ; \mathbf{w} \in W(X)\}$, thus $\kappa(X)$ is maximal order of a critical fragment for $X$.

A particular role is played by spaces $X \subseteq\{0,1\}^{K}$ for which $\kappa(X)=2$; these are known as median spaces in combinatorial mathematics. Their importance in the theory of aggregation has been emphasized by Barthélémy and Monjardet (1981) and Nehring and Puppe $(2007,2010)$. Note that a set of feasible views $X$ is a median space if and only if all logical interrelations are confined to simple implications: for some $j, k$ and all $\mathbf{x} \in X$, $x_{j}=0$ implies that $x_{k}=0$, or $x_{j}=0$ implies that $x_{k}=1$. The simplest example of a median space is the line considered in Example 1.1 above.

Proposition 1.9 Let $X \subseteq\{0,1\}^{K}$. The following statements are equivalent: [i] $X$ is path-independent; [ii] $X$ is majority determinate; [iii] $X$ is a median space, i.e. $\kappa(X)=2$.

\footnotetext{
${ }^{12}$ Note that we do not claim that all Condorcet admissible views for a given profile are equally satisfactory from a normative point of view. To the contrary, in many cases there can be additional considerations that, while not entailed by majoritarianism per se, privilege some Condorcet admissible views over others, see Nehring and Pivato (2012).
} 


\subsection{Indeterminacy and Path-Dependence}

By Proposition 1.9, the Condorcet set does not determine a unique collective view in most cases, and by Lemma 1.5 it then consists of at least three elements. In particular, in these cases there exist issues that are indeterminate in the sense that either answer in these issues is compatible with Condorcet admissibility. Accordingly, for $X \subseteq\{0,1\}^{K}$ and $\mu \in \Delta^{*}(X)$, say that issue $k \in[1 \ldots K]$ is indeterminate if there exist $\mathbf{x}, \mathbf{y} \in \operatorname{Cond}(X, \mu)$ such that $x_{k} \neq y_{k}$. We denote the set of all indeterminate issues at $\mu$ by $\operatorname{Indet}(\mu)$. Thus, $\operatorname{Indet}(\mu)$ is the set of issues in which either answer is compatible with the principle of majoritarianism as embodied in the notion of Condorcet admissibility. Diachronically speaking, $\operatorname{Indet}(\mu)=\left\{k \in[1 \ldots K]\right.$; there exist paths $\gamma$ and $\zeta$ such that $\left.F_{k}^{\gamma}(\mu) \neq F_{k}^{\zeta}(\mu)\right\}$ (by Proposition 1.8). The issues in $[1 \ldots K] \backslash \operatorname{Indet}(\mu)$ are called the determinate issues. Finally, we say that $\mu$ is globally indeterminate if $\operatorname{Indet}(\mu)=[1 \ldots K]$, and we say that $X$ is globally indeterminate if there exists some globally indeterminate $\mu \in \Delta^{*}(X)$.

We turn now to our main results, the general characterizations of indeterminacy and global indeterminacy for all aggregation spaces.

\section{Indeterminacy and Global Indeterminacy: A General Characterization}

Consider any unconstrained view $\mathbf{x} \in\{0,1\}^{K}$ and any aggregation space $X \subseteq\{0,1\}^{K}$. Say that $\mathbf{x}$ activates the critical fragment $\mathbf{w}$ if $\mathbf{x}$ coincides with $\mathbf{w}$ on its support, i.e. $\mathbf{x}_{\operatorname{supp}(\mathbf{w})}=$ $\mathbf{w}$. Furthermore, say that $\mathbf{x}$ activates issue $k$ if it activates some critical fragment $\mathbf{w}$ such that $k \in \operatorname{supp}(\mathbf{w})$. Lastly, say that the profile $\mu$ activates a critical fragment, respectively an issue, if the corresponding issue-wise majority view $\operatorname{Maj}(\mu)$ does.

Theorem 2.1 Let $X \subseteq\{0,1\}^{K}$ be any aggregation space and let $\mu \in \Delta^{*}(X)$ be any profile. Then issue $k$ is indeterminate if and only if $k$ is activated by $\mu$.

The proof of Theorem 2.1 in the appendix proceeds as follows. If an issue $k$ is indeterminate, then there exist Condorcet admissible views $\mathbf{x}$ and $\mathbf{y}$ such that $x_{k} \neq y_{k}$. One of these views, say $\mathbf{x}$, must disagree with the majority view on issue $k$. Proposition 1.8 says that there is some path $\zeta$ such that $F^{\zeta}(\mu)=\mathbf{x}$. Through an inductive construction, we show that $F^{\zeta}(\mu)$ disagrees with the majority view on issue $k$ if and only if the majority view contains a critical fragment which involves issue $k$, and such that $\zeta$ encounters (and $F^{\zeta}(\mu)$ agrees with) every other issue of this critical fragment before $\zeta$ reaches $k$, and is thus forced to disagree with the majority on issue $k$ (see Proposition A.4 in the appendix). This implies that every indeterminate issue is involved in some critical fragment activated by $\mu$. Conversely, given any critical fragment activated by $\mu$, and any issue $k$ involved in that fragment, we can construct a path $\zeta$ which traverses the rest of the critical fragment before reaching $k$, and thus must contradict the majority view in $k$. This shows that $k$ is indeterminate.

Theorem 2.1 yields a simple combinatorial characterization of globally indeterminate profiles and spaces, as follows. Say that an unconstrained view $\mathbf{x}$ is critical for $X$ if $\mathbf{x}$ 
activates every issue in $[1 \ldots K]$, and denote the set of all views that are critical for $X$ by $\operatorname{Crit}(X)$.

Theorem 2.2 Let $X \subseteq\{0,1\}^{K}$.

(a) For any $\mu \in \Delta^{*}(X),(\mu$ is globally indeterminate $) \Longleftrightarrow(\operatorname{Maj}(\mu) \in \operatorname{Crit}(X))$.

(b) $(X$ is globally indeterminate $) \Longleftrightarrow(\operatorname{Maj}(X) \cap \operatorname{Crit}(X) \neq \emptyset)$.

Proof: (a) follows immediately from Theorem 2.1, and (b) follows from (a).

Thus, a profile is globally indeterminate if and only if the majority ideal point at this profile is critical for $X$, and the space $X$ itself is globally indeterminate if and only if it gives rise to a majority ideal point that is critical for $X$. For detailed illustration in paradigmatic applications, see Section 3 below.

To apply Theorem 2.2(b) to show that a space $X$ is globally indeterminate, one needs to find an unconstrained view that is critical for $X$ and that is the issue-wise majority view for some profile $\mu$ on $X$. To determine whether such a profile exists, for any given view, may be difficult in general, hence Theorem 2.2 may be difficult to apply. However, in a significant number of cases, the McGarvey spaces, this problem does not arise, as these spaces are defined by the property that $\operatorname{Maj}(X)=X$; see Nehring and Pivato (2011) for a detailed analysis and numerous examples, including the spaces $X_{N}^{\mathrm{pr}}$ and $X_{N}^{\mathrm{eq}}$ corresponding to preference aggregation and the classification problem (see Sections 3.1 and 3.2 below).

Theorem 2.1 also entails a characterization of when no issue is indeterminate given a profile, i.e. of when issue-wise majority voting is consistent for a given profile.

Corollary 2.3 The profile $\mu$ is majority determinate if and only if $\mu$ does not activate any critical fragment.

The question of majority determinacy of a given profile in an arbitrary aggregation space has been addressed before by Dietrich and List (2010) and Pivato (2009). The condition derived in Corollary 2.3 readily translates into Dietrich and List's (2010) condition of 'majority consistency.' Dietrich and List (2010) also provide a number of other, simpler but only sufficient conditions for majority determinacy of a given profile, among them the counterpart in the general judgement aggregation model of Sen's classical condition of 'value restriction' (see Sen (1970)). Pivato (2009) provides a class of sufficient geometric conditions for determinacy of a profile.

Theorem 2.1 also yields via Corollary 2.3 the characterization of majority determinate spaces as median spaces stated in Proposition 1.9. This follows from the observation that a critical fragment $\mathbf{w}$ of $X$ is activated by some profile $\mu \in \Delta^{*}(X)$ if and only if $\# \mathbf{w} \geq 3$. 


\section{The Condorcet Set in Paradigmatic Aggregation Problems}

In this section we will illustrate the notions of Condorcet admissibility and Condorcet set in four paradigmatic examples: preference aggregation, the aggregation of equivalence relations (the "classification problem"), committee selection and the allocation of public goods.

\subsection{Preference aggregation}

Consider a finite set of $N$ social alternatives. Let $K:=N(N-1) / 2$, and bijectively identify $[1 \ldots K]$ with a subset of $[1 \ldots N] \times[1 \ldots N]$ which contains exactly one element of the set $\{(a, b),(b, a)\}$ for each distinct $a, b \in[1 \ldots N]$. Then $\{0,1\}^{K}$ represents the space of all tournaments (i.e. complete, irreflexive, antisymmetric binary relations, or equivalently, complete directed graphs) on $[1 \ldots N]$. Intuitively, each issue corresponds to a proposition of the form 'alternative $a$ is strictly preferred to alternative $b .{ }^{13}$ Let $X_{N}^{\mathrm{pr}} \subset\{0,1\}^{K}$ be the set

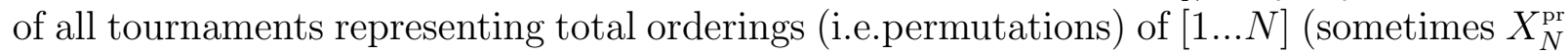
is called the permutahedron). Classical Arrovian aggregation of strict preference orderings is simply judgement aggregation on $X_{N}^{\mathrm{pr}}$. For any profile $\mu \in \Delta^{*}\left(X_{N}^{\mathrm{pr}}\right)$, the set Cond $\left(X_{N}^{\mathrm{pr}}, \mu\right)$ is the set of preference orderings on $[1 \ldots N]$ such that no other ordering agrees with the $\mu$-majority on a larger set of pairwise comparisons.

Note that in the case of preference aggregation, Condorcet admissibility always restricts the set of admissible elements, i.e. Cond $\left(X_{N}^{\mathrm{pr}}, \mu\right)$ is never all of $X_{N}^{\mathrm{pr}}$. For example, if a majority of voters strictly prefer $a$ to $b$ in the profile $\mu$, then no element of Cond $\left(X_{N}^{\mathrm{pr}}, \mu\right)$ can place $a$ and $b$ as nearest neighbours with $b \succ a$, because switching the social ranking between $a$ and $b$ while retaining all other comparisons would agree with the majority view on a strictly larger set of issues. ${ }^{14}$ In particular, the top-ranked alternative of a Condorcet admissible ordering is always preferred by a majority to the second-ranked alternative. Thus, in any Condorcet admissible ordering, the top alternatives is the majority winner in a runoff with the top two choices.

Observe also that majority determinacy in the sense defined above is stronger than the usual notion of existence of a Condorcet winner. Indeed, majority determinacy in our sense requires the existence of an entire ordering that agrees with the majority judgement in each binary comparison; it is thus equivalent to the existence of a Condorcet winner on each subset of $[1 \ldots N]$.

For any $\mathbf{x} \in\{0,1\}^{K}$, let $\stackrel{\mathbf{x}}{\succ}$ be the binary relation on $[1 \ldots N]$ defined by $\mathbf{x}$. Moreover, for any $\mu \in \Delta^{*}\left(X_{N}^{\mathrm{pr}}\right)$, let $\succ^{\mu}$ be the binary relation defined by $\operatorname{Maj}(\mu)$ - the so-called majority tournament. An element $\mathbf{y} \in X_{N}^{\mathrm{pr}}$ is called a directed Hamiltonian chain of $\operatorname{Maj}(\mu)$ if all nearest-neighbour comparisons in $\succ^{\mathbf{y}}$ agree with the orderings specified by $\succ^{\mu}$. In other

\footnotetext{
${ }^{13}$ The focus on strict orderings is for expositional convenience here. The analysis can be generalized without difficulty to the case of binary relations with non-trivial indifferences; in that case, one needs to introduce both issues $(a, b)$ (corresponding to ' $a \succeq b$ ') and $(b, a)$ (corresponding to ' $b \succeq a$ '), see Nehring et al. (2013) for a corresponding model.

${ }^{14} a \succ b$ is a nearest-neighbour comparison if for all $c$ with $a \succ c$ and $c \succ b$ either $c=a$ or $c=b$.
} 
words, if we represent $\succ^{\mathbf{y}}$ as a linear directed graph $C$ and represent $\succ^{\mu}$ as a complete directed graph $D$ in the obvious way, then $C$ is a (directed) subgraph of $D$.

Let $\succeq_{*}^{\mathbf{x}}$ be the transitive closure of $\succ^{\mathbf{x}},{ }^{15}$ augmented by all pairs $(a, a)$ for $a \in[1 \ldots N]$; then $\succeq_{*}^{\mathbf{x}}$ is a weak order (i.e.it is complete, reflexive and transitive). The symmetric part $\stackrel{\mathrm{x}}{\approx}_{*}$ of $\stackrel{\succeq}{*}_{*}$ is an equivalence relation (one has $a \stackrel{\mathrm{x}}{\approx}_{*} b$ iff ' $a$ and $b$ belong to the same cycle of $\left.\stackrel{x}{\succ}^{\mathbf{x}}\right)$. The $\stackrel{\mathrm{x}}{\approx}_{*}$-equivalence classes of $[1 \ldots N]$ are linearly ordered by the asymmetric part $\succ_{*}^{\mathrm{x}}$

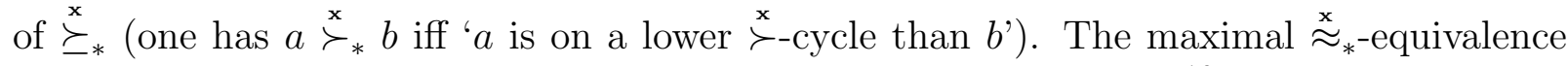
class is also known as the top cycle of the tournament defined by $\mathbf{x}^{16}$

Proposition 3.1 Let $\mu \in \Delta^{*}\left(X_{N}^{\mathrm{pr}}\right)$.

(a) Cond $\left(X_{N}^{\mathrm{pr}}, \mu\right)=\left\{\mathbf{x} \in X_{N}^{\mathrm{pr}} ; \stackrel{\mathrm{x}}{\succ}\right.$ is a directed Hamiltonian chain in $\stackrel{\mu}{\succ}^{\prime}$.

(b) For all $a, b \in[1 \ldots N], a \stackrel{\mu}{\succ}_{*} b$ if and only if, for all $\mathbf{x} \in \operatorname{Cond}\left(X_{N}^{\mathrm{pr}}, \mu\right), a \stackrel{\mathrm{x}}{\succ} b$. In particular, the determinate issues given a profile $\mu$ are exactly the issues $(a, b)$ for which either $a \stackrel{\mu}{\succ}_{*} b$, or $b \stackrel{\iota}{\succ}_{*} a$.

(c) The profile $\mu$ is globally indeterminate if and only if the top cycle of $\succ^{\mu}$ equals $[1 \ldots N]$. (Such profiles exist.)

While directed Hamiltonian chains are a well-known object in combinatorics, their characterization in Proposition 3.1(a) appears to be new in the social choice literature. Note also that, by Proposition 3.1(b), for every profile $\mu$, the top cycle set of the majority tournament consists exactly of the top elements of the Condorcet admissible orderings.

Example 3.2 (Condorcet Set in the 4-permutahedron) Consider $X_{N}^{\mathrm{pr}}$ with the set of alternatives $N=\{a, b, c, d\}$. Suppose that one third of the population endorses each of the preference orderings $a \succ b \succ c \succ d, b \succ c \succ d \succ a$ and $c \succ d \succ a \succ b$. For the corresponding majority tournament we have $c \succ^{\mu} a, d \succ^{\mu} a, a \succ^{\mu} b, b \succ^{\mu} c, b \succ^{\mu} d$, and $c \succ^{\mu} d$ (see Fig. 2). By Proposition 3.1(a), the Condorect set consists of the following five orderings: $a \succ b \succ c \succ d, b \succ c \succ d \succ a, c \succ d \succ a \succ b, d \succ a \succ b \succ c, c \succ a \succ b \succ d$. The global indeterminacy of the considered profile follows from Proposition 3.1(c).

By Proposition 3.1(b), the top cycle set of a majority tournament characterizes the choice implications of 'majoritarian' preference aggregation: an alternative is in the top cycle if and only if it is on top of some Condorcet admissible ordering. While choice from the top cycle of the underlying majority tournament is thus a necessary condition for Condorcet admissibility, it is by no means sufficient. Indeed, as already noted, Condorcet admissibility entails additional restrictions that do not only concern the top elements. The following example provides a further illustration.

\footnotetext{
${ }^{15}$ By definition, the pair $(a, b)$ belongs to the transitive closure of $\succ$ if there exist $a_{1}, \ldots, a_{m}$ such that $a=a_{1}, b=a_{m}$ and $a_{j} \succ a_{j+1}$ for all $j=1, \ldots, m-1$.

${ }^{16}$ The concept of the top cycle was introduced by Good (1971), see also (Moulin, 1988, p.253). For a choice-theoretic analysis of the relation $\stackrel{x}{*}_{*}$ see Duggan (2007).
} 


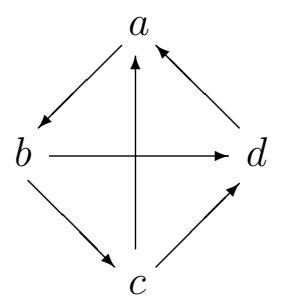

Figure 2: A majority tournament on four alternatives

Example 3.3 (The 'Fairest Vote of All') The Copeland rule ranks each alternative according to the number of alternatives which it beats in pairwise majority comparisons, and selects the alternative(s) with the largest such number. For instance, in Fig. 2, the alternatives $b$ and $c$ each receive a 'Copeland score' of 2, while $a$ and $d$ receive a score of 1 ; thus, the 'Copeland winners' are $b$ and $c$. Evidently, the Copeland rule chooses a Condorcet winner whenever it exists. As seen from this simple example, however, the Copeland rule often produces ties. To solve this problem, Dasgupta and Maskin (2004) have proposed the following refinement of the Copeland rule under the name of the 'fairest vote of all. ${ }^{17}$ First, order alternatives according to their Copeland score; if this yields a tie between two or more alternatives, order those according to their Borda scores while retaining the lexicographic precedence of the Copeland score among the other alternatives (note that the Borda rule generically yields a strict ranking of alternatives). Evidently, the resulting voting rule chooses from the top cycle; however, it does in general not produce a Condorcet admissible ranking. For instance, for the profile in Example 3.2 it yields the unique ordering $c \succ b \succ a \succ d$ which is not Condorcet admissible because in the case of both nearest-neighbour pairs $(c, b)$ and $(a, d)$ a majority prefers the lower ranked alternative. We thus conclude that the method proposed by Dasgupta and Maskin is not 'genuinely' majoritarian; this makes intuitive sense, since the invocation of Borda count introduces prima facie non-majoritarian considerations.

\subsection{Classification}

An interesting example of judgement aggregation frequently discussed in the literature is the 'classification problem,' i.e. the problem of aggregating a set of equivalence relations (see Fishburn and Rubinstein (1986) and, for a recent contribution, Dimitrov et al. (2012)). The individual equivalence relations correspond to the classifications of a set of objects by different experts. The experts disagree about the appropriate classification into equivalence classes, and the problem consists in finding an overall classification that best represents their view as a group.

Formally, let $N \in \mathbb{N}$, let $K:=N(N-1) / 2$, and identify $[1 \ldots K]$ with a subset of $[1 \ldots N] \times[1 \ldots N]$ containing exactly one of the pairs $(a, b)$ or $(b, a)$ for each $a \neq b \in[1 \ldots N]$.

\footnotetext{
${ }^{17}$ Originally, Dasgupta and Maskin (2004) proposed it as a voting rule, i.e. an aggregation method that specifies only a chosen alternative given any profile of individual preferences; we present here the straightforward extension of Dasgupta's and Maskin's method to a preference aggregation rule.
} 
Thus, an element of $\{0,1\}^{K}$ may represent a symmetric, reflexive binary relation, i.e. an undirected graph on $[1 \ldots N]$. By $X_{N}^{\text {eq }} \subseteq\{0,1\}^{K}$ we denote the set of all equivalence relations on $[1 \ldots N]$. The equivalence relation represented by $\mathbf{x} \in X_{N}^{\text {eq }}$ will be denoted by $\stackrel{\mathbf{x}}{\sim}$; moreover, for any graph $\mathbf{x} \in\{0,1\}^{K}$ denote by $\operatorname{tr}(\mathbf{x})$ its transitive closure. The graph $\mathbf{1}$ is called the complete graph; $\mathbf{x}$ is called connected if $\operatorname{tr}(\mathbf{x})=\mathbf{1}$. The following result characterizes the Condorcet set and the determinate issues for every profile in the classification problem.

Proposition 3.4 Let $\mu \in \Delta^{*}\left(X_{N}^{\mathrm{eq}}\right)$, and let $\mathrm{x}^{\mu} \in\{0,1\}^{K}$ be the issue-wise majority judgement (the 'majority graph').

(a) Let $\mathbf{x} \in X_{N}^{\mathrm{eq}}$ represent the equivalence relation $\stackrel{\mathbf{x}}{\sim}$ on $N$. Then, $\mathbf{x} \in \operatorname{Cond}\left(X_{N}^{\mathrm{eq}}, \mu\right)$ if and only if: [i] $\stackrel{\mathbf{x}}{\sim} \subseteq \operatorname{tr}\left(\mathbf{x}^{\mu}\right)$, and [ii] if not $a \stackrel{\mathbf{x}}{\sim} b$, then there exist $a^{\prime}, b^{\prime} \in N$ such that $a \stackrel{\mathbf{x}}{\sim} a^{\prime}\left(\right.$ or $\left.a=a^{\prime}\right), b \stackrel{\mathbf{x}}{\sim} b^{\prime}\left(\right.$ or $\left.b=b^{\prime}\right)$, and $\left\{a^{\prime}, b^{\prime}\right\}$ is not an edge of $\mathbf{x}^{\mu}$.

(b) The issue $(a, b) \in[1 \ldots K]$ is determinate if and only if $\mathbf{x}_{(a, b)}^{\mu}=0$ and $\operatorname{tr}\left(\mathbf{x}^{\mu}\right)_{(a, b)}=$ 0 , or $\mathbf{x}_{(a, b)}^{\mu}=1$ and $\operatorname{tr}\left(\mathbf{x}^{\mu}\right)_{(c, d)}=\mathbf{x}_{(c, d)}^{\mu}=1$ for all edges $(c, d) \in[1 \ldots K]$ belonging to the same connected component of $\mathbf{x}^{\mu}$ as $(a, b)$.

(c) The profile $\mu$ is globally indeterminate if and only if $\mathbf{x}^{\mu}$ is connected but not complete. (Such profiles exist.)

Proposition 3.4(c) is illustrated in the case of $N=3$ in Fig. 3. The top graph is the majority graph $\mathbf{x}^{\mu}$ of the profile $\mu$ that puts equal popular weight to the following three equivalence relations, represented by their corresponding partitions of $\{a, b, c\}$, respectively: $\mathbf{x}_{1}$ corresponding to $\{\{a, b, c\}\}, \mathbf{x}_{2}$ corresponding to $\{\{a, b\},\{c\}\}$, and $\mathbf{x}_{3}$ corresponding $\{\{b\},\{a, c\}\}$. The Condorcet set is given by $\left\{\mathbf{x}_{1}, \mathbf{x}_{2}, \mathbf{x}_{3}\right\}$ (see the three graphs at the bottom in Fig. 3); global indeterminacy is easily verified.

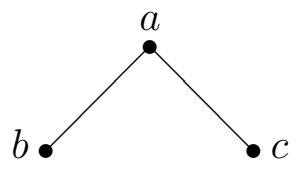

The majority ideal
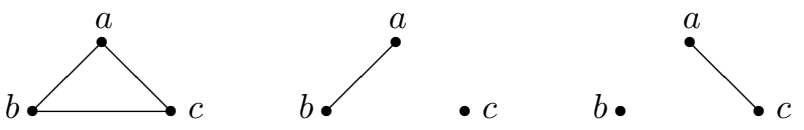

The Condorcet set

Figure 3: Global indeterminacy in the classification problem 


\subsection{Committee selection}

For any $\mathbf{x} \in\{0,1\}^{K}$, let $\|\mathbf{x}\|:=\#\left\{k \in[1 \ldots K] ; x_{k}=1\right\}$. Let $0 \leq I \leq J \leq K$, and define

$$
X_{I, J ; K}^{\mathrm{com}}:=\quad\left\{\mathbf{x} \in\{0,1\}^{K} ; I \leq\|\mathbf{x}\| \leq J\right\} .
$$

Heuristically, $[1 \ldots K]$ is a set of $K$ 'candidates', and $X_{I, J ; K}^{\text {com }}$ is the set of all 'committees' comprised of at least $I$ and at most $J$ of these candidates. ${ }^{18}$ The characterization of the Condorcet set in this case follows immediately from Lemma 1.5:

Proposition 3.5 Let $0 \leq I \leq J \leq K$ and let $\mu \in \Delta^{*}\left(X_{I, J ; K}^{\text {com }}\right)$. Let $M:=\{k \in[1 \ldots K]$; $\left.\mu\left(x_{j}\right)>\frac{1}{2}\right\}$ be the set of all candidates receiving majority support.

(a) If $I \leq|M| \leq J$, then Cond $\left(X_{I, J ; K}^{\mathrm{com}}, \mu\right)=\left\{\mathbf{1}_{M}\right\}$.

(b) If $|M|<I$, then Cond $\left(X_{I, J ; K}^{\mathrm{com}}, \mu\right)=\left\{\mathbf{1}_{H} ; M \subset H \subseteq[1 \ldots K]\right.$, and $\left.|H|=I\right\}$.

(c) If $|M|>J$, then Cond $\left(X_{I, J ; K}^{\mathrm{com}}, \mu\right)=\left\{\mathbf{1}_{H} ; H \subset M\right.$ and $\left.|H|=J\right\}$.

Next, we study indeterminacy in the committee selection problem. The set of (in)determinate issues given any profile $\mu$ is easily determined using Proposition 3.5. In contrast to the preference aggregation and classification problems, the results concerning global indeterminacy are multifaceted in the committee selection problem. While global indeterminacy again occurs often, it does not occur always.

Proposition 3.6 Consider $X_{I, J ; K}^{\mathrm{com}}$ and suppose that $I>0$ and $J<K$. Then $X_{I, J ; K}^{\mathrm{com}}$ is globally indeterminate unless $I=J=K / 2$.

The restriction to the case $I>0$ and $J<K$ is essential for the conclusion of Proposition 3.6. Indeed, otherwise the committee selection problem displays the following property of 'comprehensiveness' which leads to significantly different results. For any $\mathbf{x}, \mathbf{y} \in\{0,1\}^{K}$, write $\mathbf{x} \leq \mathbf{y}$ if $x_{k} \leq y_{k}$ for all $k \in[1 \ldots K]$. A subset $X \subset\{0,1\}^{K}$ is comprehensive if, for any $\mathbf{x}, \mathbf{y} \in\{0,1\}^{K}$, if $\mathbf{x} \in X$ and $\mathbf{x} \leq \mathbf{y}$, then also $\mathbf{y} \in\{0,1\}^{K}$ also. Thus, a space is comprehensive if any view affirming a larger set of propositions than some feasible view is feasible as well. For example, $X_{I, K ; K}^{\text {com }}$ is comprehensive, for any $I \leq K{ }^{19}$

In the following result, a space $X$ is called non-degenerate if, for every $k \in[1 \ldots K]$, there is some critical fragment $\mathbf{w}$ of order at least 3 such that $k \in \operatorname{supp}(\mathbf{w})$. For example, the space $X_{K-1, K ; K}^{\mathrm{com}}$ is degenerate since all critical fragments have order $2 .{ }^{20}$

Proposition 3.7 Let $X$ be comprehensive and non-degenerate. Then $X$ is globally indeterminate if and only if $\mathbf{0} \in \operatorname{Maj}(X)$.

\footnotetext{
${ }^{18}$ The committee selection problem has a long history in social choice theory; according to McLean (1990) the aggregation problem corresponding to $X_{7,7 ; 20}^{\mathrm{com}}$ was studied by Ramon Lull already in the year 1274 in his 'Book of the Gentile and the Three Wise Men.' For a related model interpreted in terms of 'community standards,' see Miller (2009).

${ }^{19}$ Likewise, the spaces $X_{0, J ; K}^{\text {com }}$ for $0 \leq J \leq K$ are isomorphic to comprehensive problems.

${ }^{20}$ It follows from the analysis in $\S 2$ above that degenerate spaces are never globally indeterminate.
} 
To illustrate, consider the class of comprehensive committee selection problems $X_{I, K ; K}^{\text {com }}$. Here, Proposition 3.7 yields the following corollary.

Corollary 3.8 Let $I>0$. The space $X_{I, K ; K}^{\mathrm{com}}$ is globally indeterminate if and only if $I<$ $K / 2$.

To verify this, simply observe that $\mathbf{0} \in \operatorname{Maj}\left(X_{I, K ; K}^{\text {com }}\right)$ only if $I<K / 2$; indeed, if every voter approves of more than half of the candidates, then at least one candidate must receive a majority of votes.

\subsection{Resource allocation}

In this subsection, we show how the general judgement aggregation framework allows one to represent and analyze resource allocation problems. While global indeterminacy can also arise in this context, the indeterminacy turns out to be much more 'well-behaved' than in the cases of preference aggregation or classification.

Fix $M, Q \in \mathbb{N}$, and consider the $Q$-dimensional 'discrete cube' $[0 \ldots M]^{Q}$. Each element $\mathbf{x} \in[1 \ldots M]^{Q}$ can be represented by a point $\Phi(\mathbf{x}):=\widetilde{\mathbf{x}} \in\{0,1\}^{Q \times M}$ defined as follows:

$$
\text { for all }(q, m) \in[1 \ldots Q] \times[1 \ldots M], \quad \widetilde{x}_{(q, m)}:= \begin{cases}1 & \text { if } \quad x_{q} \geq m \\ 0 & \text { if } \quad x_{q}<m\end{cases}
$$

For example, $\Phi\left(\mathbf{0}^{Q}\right)=\mathbf{0}^{Q \times M}$ and $\Phi\left(\mathbf{1}^{Q}\right)=\mathbf{1}^{Q \times M}$. This defines an injection $\Phi:[0 \ldots M]^{Q}$ $\longrightarrow\{0,1\}^{Q \times M}$. Any subset of $P \subseteq[0 \ldots M]^{Q}$ can thereby be represented as a subset $X:=\Phi(P) \subseteq\{0,1\}^{Q \times M}$. Judgement aggregation over $X$ thus represents social choice over a $Q$-dimensional 'policy space', where each voter's position represents her ideal point in $P$, the set of feasible policies. This framework is especially useful for resource allocation problems, as we now illustrate. Let

$$
\begin{aligned}
\triangle_{M}^{Q} & :=\left\{\mathrm{x} \in[0 \ldots M]^{Q} ; \sum_{q=1}^{Q} x_{q}=M\right\}, \\
\text { and } X_{M, Q}^{\Delta} & :=\Phi\left[\triangle_{M}^{Q}\right] \subseteq\{0,1\}^{Q \times M} .
\end{aligned}
$$

Geometrically, $\triangle_{M}^{Q}$ is a 'discrete simplex'; points in $\triangle_{M}^{Q}$ represent all ways of allocating $M$ indivisible dollars among $Q$ public goods. Thus, judgement aggregation over $X_{M, Q}^{\Delta}$ describes a group which decides how to allocate a budget of $M$ dollars to $Q$ public goods by voting 'yea' or 'nay' to propositions of the form ' $x_{q}$ should be at least $m$ dollars' for each $q \in[1 \ldots Q]$ and $m \in[1 \ldots M]$. We will thus henceforth refer to spaces of the form $X_{M, Q}^{\Delta}$ as budget spaces, cf. Lindner et al. (2010).

On the space $X_{M, Q}^{\Delta}$, the Condorcet set allows for the following explicit characterization. For each $q \in[1 \ldots Q]$ and every $\mu \in \Delta^{*}\left(X_{M, Q}^{\Delta}\right)$, let $m_{q}^{*}:=\operatorname{med}_{q}(\mu)$ denote the median in coordinate $q$ (that is: $m_{q}^{*}$ is the unique $m \in[0 \ldots M]$ such that $\left.\mu\left(x_{q, m}\right)>\frac{1}{2}>\mu\left(x_{q, m+1}\right)\right\}$; as already noted this value exists because $\left.\mu \in \Delta^{*}\left(X_{M, Q}^{\Delta}\right)\right)$. It follows that $\operatorname{Maj}(\mu)=$ $\Phi\left(m_{1}^{*}, \ldots, m_{Q}^{*}\right)$ (see Lemma A.3(b)). Let $D(\mu):=\left(\sum_{q=1}^{Q} m_{q}^{*}\right)-M$ be the "majority deficit' corresponding to the profile $\mu$. Note that the majority deficit can be positive or negative. 
Proposition 3.9 Let $M, Q \in \mathbb{N}$, and let $\mu \in \Delta^{*}\left(X_{M, Q}^{\Delta}\right)$.

(a) If $D(\mu)=0$, then Cond $\left(X_{M, Q}^{\Delta}, \mu\right)=\operatorname{Maj}(\mu)$.

(b) If $D(\mu)>0$, then Cond $\left(X_{M, Q}^{\Delta}, \mu\right)=\left\{\Phi(\mathbf{x}) ; \mathbf{x} \in \triangle_{M}^{Q}\right.$ and $x_{q} \in\left[m_{q}^{*}-D(\mu), m_{q}^{*}\right]$ for all $q \in[1 \ldots Q]\}$.

(c) If $D(\mu)<0$, then Cond $\left(X_{M, Q}^{\Delta}, \mu\right)=\left\{\Phi(\mathbf{x}) ; \mathbf{x} \in \triangle_{M}^{Q}\right.$ and $x_{q} \in\left[m_{q}^{*}, m_{q}^{*}+|D(\mu)|\right]$ for all $q \in[1 \ldots Q]\}$.

In particular, one has Cond $\left(X_{M, Q}^{\Delta}, \mu\right)=$ Slater $\left(X_{M, Q}^{\Delta}, \mu\right)$ for all $\mu$.

Thus, a profile $\mu$ is majority determinate if and only if $D(\mu)=0$. Moreover, if there is positive (negative) deficit, the Condorcet set arises by allocating at most (at least) the median amount to each claimant while distributing the slack in any feasible way.

Note that, if $Q=2$, the spaces $X_{M, Q}^{\Delta}$ have the structure of a line, in particular $X_{M, Q}^{\Delta}$ is a median space in this case, hence majority determinate. The following result characterizes global indeterminacy in the spaces $X_{M, Q}^{\Delta}$ if $Q \geq 3$ and describes the qualitative structure of indeterminacy in these spaces in general. For any profile $\mu$ define the degree of indeterminacy $\operatorname{ind}(\mu)$ as the number of indeterminate issues given $\mu$, i.e.

$$
\operatorname{ind}(\mu):=\# \operatorname{Indet}(\mu) \text {. }
$$

Proposition 3.10 Consider a budget space $X_{M, Q}^{\Delta}$ and suppose that $Q \geq 3$.

(a) A profile $\mu$ is globally indeterminate if and only if $m_{q}^{*}=0$ for all $q \in[1 \ldots Q]$.

(b) For all profiles $\mu$, ind $(\mu) \leq Q \cdot|D(\mu)|$.

Note that, if $Q \geq 3, m_{q}^{*}(\mu)=0$ for all $q \in[1 \ldots Q]$ can easily occur; for instance, this will be the case if there are sufficiently many voters and every voter is only interested in positive quantities of less than half of the goods $q \in[1 \ldots Q]$ (provided that the relevant sets of goods differ across voters). By Proposition 3.10(a), global indeterminacy can thus arise in nondegenerate cases in the resource allocation problem (if $Q \geq 3$ ). On the other hand, Proposition 3.10(b) shows that typical profiles lead to 'local' but not global indeterminacy. In particular, the indeterminacy will not be global whenever every agent's ideal allocation involves a positive amount of more than half of the goods, as stated by the following result.

Corollary 3.11 Consider a budget space $X_{M, Q}^{\Delta}$ and suppose that $\mu \in \Delta^{*}\left(X_{M, Q}^{\Delta}\right)$ is such that every agent's allocation involves a positive quantity of more than half of the goods. Then, $\operatorname{Maj}(\mu) \neq \mathbf{0}$; in particular, by Proposition 3.10(a), $\mu$ is not globally indeterminate. 


\section{When Does Majority Voting Lead to Chaos? The 'McKelvey' Criterion}

Consider again the majority tournament shown in Fig. 2 above. As we have seen, any profile that induces this majority tournament is globally indeterminate. On the other hand, the displayed majority tournament is 'nearly' consistent: one only needs to reverse the binary comparison between $a$ and $b$ to obtain the consistent (transitive) ordering $b \succ c \succ d \succ$ $a$. This demonstrates that just a little inconsistency can cause global indeterminacy in the preference aggregation problem, an observation strongly reminiscent of McKelvey's classical 'chaos' theorem in a spatial context, see McKelvey (1979).

Similarly, consider a graph $\mathbf{x} \in\{0,1\}^{K}$ that partitions the set $[1 \ldots N]$ into two cliques $A_{1}$ and $A_{2}$ and that contains exactly one additional edge connecting some $a \in A_{1}$ with some $b \in A_{2}$. By Proposition 3.4(c), any profile in $\Delta^{*}\left(X_{N}^{\mathrm{eq}}\right)$ that induces $\mathbf{x}$ as the issuewise majority judgement is globally indeterminate. But as above, among all elements in $\{0,1\}^{K} \backslash X_{N}^{\mathrm{eq}}, \mathbf{x}$ is as close as possible to $X_{N}^{\mathrm{eq}}$ in the sense that the value in only one issue has to be reversed in order to obtain an equivalence relation (by removing the edge $(a, b))$. Thus, also in the classification problem a little inconsistency can cause global indeterminacy. This motivates the following definitions.

For any judgement aggregation space $X \subseteq\{0,1\}^{K}$ and any $\mathbf{y} \in\{0,1\}^{K}$ define the degree of inconsistency of $\mathbf{y}$ (relative to $X$ ) as

$$
\operatorname{inc}(\mathbf{y}):=\min _{\mathbf{x} \in X} d_{H}(\mathbf{x}, \mathbf{y})
$$

Also, for any profile $\mu$, let

$$
\operatorname{inc}(\mu):=\operatorname{inc}[\operatorname{Maj}(\mu)]=\min _{\mathbf{x} \in X} d_{H}[\mathbf{x}, \operatorname{Maj}(\mu)] \cdot{ }^{21}
$$

Say that an aggregation space $X$ is McKelvey if there exists a globally indeterminate profile $\mu \in \Delta^{*}(X)$ such that inc $(\mu)=1$. Thus, a space is McKelvey if there exists a globally indeterminate profile such that its corresponding issue-wise majority view is only minimally inconsistent. Any feasible view $\mathbf{x} \in X$ that verifies the McKelvey property, i.e. that satisfies $d_{H}[\mathbf{x}, \operatorname{Maj}(\mu)]=1$ for some globally indeterminate profile $\mu$, will be referred to as a McKelvey view.

Theorem 2.2(a) entails the following straighforward characterization of McKelvey spaces.

Corollary 4.1 A space $X \subseteq\{0,1\}^{K}$ is McKelvey if and only if there exists a profile $\mu$ such that $\operatorname{Maj}(\mu) \in \operatorname{Crit}(X) \cap X^{\prime}$ where

$$
X^{\prime}:=\left\{\mathbf{y} \in\{0,1\}^{K} \backslash X ; \min _{\mathbf{x} \in X} d_{H}(y, x)=1\right\} .
$$

\footnotetext{
${ }^{21}$ In the context of preference aggregation, this 'inconsistency measure' was first proposed by Slater (1961). As pointed out by (Laslier, 1997, p.68), Slater was in fact more interested in the measure itself than in the now called 'Slater rule' that selects, for any profile $\mu$, the minimizers in $X$ of $\operatorname{inc}[\operatorname{Maj}(\mu)]$ (cf. Footnote 10 above).
} 
Above, we have seen that the preference aggregation problem is McKelvey. In fact, in the preference aggregation example every feasible view is a McKelvey view. To see this, take any preference ordering $\succ \in X_{N}^{\mathrm{pr}}$, say with $a_{1} \succ a_{2} \succ \ldots \succ a_{N}$, and consider the tournament $\mathbf{x} \in\{0,1\}^{K}$ that coincides with $\succ$ except in issue $\left(a_{1}, a_{N}\right)$ in which it specifies $a_{1} \stackrel{\mathbf{x}}{\prec} a_{N}$. By construction, we have $\operatorname{inc}(\mathbf{x})=1$. Moreover, any profile $\mu$ such that $\mathbf{x}=\operatorname{Maj}(\mu)$ is globally indeterminate by Proposition 3.1(c) ${ }^{22}$

The spaces underlying the classification problem are also McKelvey as shown above. The McKelvey views are exactly the equivalence relations that partition $[1 \ldots N]$ into two equivalence classes, see Proposition 4.2(b) below.

By contrast, resource allocation problems are never McKelvey by Proposition 3.10(a). Moreover, since inc $(\mu)=|D(\mu)|$ in this case, the degree of indeterminacy of a profile is proportional to the degree of its inconsistency by Proposition 3.10(b). Resource allocation problems are thus an interesting example of aggregation problems in which majoritarian aggregation may be highly indeterminate, yet well-behaved and 'non-chaotic.'

The extent of potential majority 'chaos' can be measured quantitatively by the following McKelvey index $\chi(X)$ of an aggregation space $X$ as the smallest constant $C \in \mathbb{R}$ such that, for all profiles $\mu \in \Delta^{*}(X)$,

$$
\operatorname{ind}(\mu) \leq C \cdot K \cdot \operatorname{inc}(\mu) .
$$

By construction, for any $X$, one has $0 \leq \chi(X) \leq 1$. At the extremes, one has $\chi(X)=1$ just in case $X$ is a McKelvey space; on the other hand, $\chi(X)=0$ just in case $X$ is majority determinate, i.e. a median space. Budget spaces with at least 3 goods occupy a middle ground. Indeed, as stated in Proposition 4.2(d) below, one obtains $\chi(X)=\frac{1}{M}$ for such spaces; thus, there is a simple quantitative sense in which majoritarian aggregation in budget spaces is far from chaotic.

The following result summarizes these findings and also shows that the committee selection problem has the McKelvey property only under restrictive conditions.

Proposition $4.2 \quad$ (a) (Preference aggregation) For $X_{N}^{\mathrm{pr}}$ one has

$$
\chi\left(X_{N}^{\mathrm{pr}}\right)=1
$$

i.e. the preference aggregation problem is McKelvey. Moreover, any feasible view in $X_{N}^{\mathrm{pr}}$ is a McKelvey view.

(b) (Classification) For $X_{N}^{\mathrm{eq}}$ one has

$$
\chi\left(X_{N}^{\mathrm{eq}}\right)=1
$$

i.e. the classification problem is McKelvey. Moreover, the McKelvey views are the equivalence relations that partition $[1 \ldots N]$ into two equivalence classes.

(c) (Committee selection) Consider $X_{I, J ; K}^{\mathrm{com}}$ and assume that $K>2$ and $I \leq J$. Then

$$
\chi\left(X_{I, J ; K}^{\mathrm{com}}\right)=1 \Longleftrightarrow(I=1 \text { or } J=K-1) .
$$

\footnotetext{
${ }^{22}$ The existence of a profile $\mu$ with $\mathbf{x}=\operatorname{Maj}(\mu)$ follows from a classic result by McGarvey (1953), see also Nehring and Pivato (2011).
} 
(d) (Resource allocation) For $X_{M, Q}^{\Delta}$ one has

$$
\chi\left(X_{M, Q}^{\Delta}\right)=\frac{1}{M},
$$

in particular, the budget spaces $X_{M, Q}^{\Delta}$ are never McKelvey.

\section{Conclusion}

We have proposed a natural and workable demarcation criterion for 'majoritarian' social choice. In particular, we challenged the view that in the absence of a Condorcet winner (or, more generally, a consistent issue-wise majority view) there is no well-defined 'will of the majority.' Indeed, in spite of the Condorcet paradox it is possible to define a consistent and generally applicable notion of 'majoritarianism,' the Condorcet set. However, this comes at the price of indeterminacy, since the Condorcet is often 'large.' In this paper, we have analyzed the structure of indeterminacy in some detail, but there remain a number of interesting open problems.

First, is it possible to refine the notion of Condorcet admissibility using quantitative information on the majority margins in each issue? This is answered positively in Nehring and Pivato (2012), by exploring a condition of symmetry across issues.

Secondly, one may ask under what circumstances the path-dependence entailed by sequential majority voting can even force one to override unanimous consent in some issues. This problem is addressed in Nehring et al. (2013), where we characterize the aggregation problems for which sequential majority voting never overrides unanimous consent no matter in which sequence the decisions are taken ('strong sequential unanimity consistency'). It turns out that this is a very restricted class, but we also give examples of interesting aggregation problems for which there exist particular decision paths along which sequential majority voting respects unanimous consent at all profiles ('weak sequential unanimity consistency').

Several further questions are worthwhile to address. For example, what is the asymptotic behavior of the Condorcet set as the number of issues grows? Or, what is the distribution over the outcomes of sequential majority voting induced by a given distribution over the set of possible decisions paths? And what is the induced probability of unanimity violations? We hope that the present paper will stimulate further research along these or similar lines.

\section{Appendix: Proofs}

Notation. We define the elements $\mathbf{0}^{K}:=(0,0, \ldots, 0)$ and $\mathbf{1}^{K}:=(1,1, \ldots, 1)$ in $\{0,1\}^{K}$ (we will simply write " 0 " and "1" when $K$ is clear from context). For any subset $J \subseteq[1 \ldots K]$, let $\mathbf{1}_{J}$ denote the vector $\mathbf{x} \in\{0,1\}^{K}$ such that $x_{j}=1$ for all $j \in J$ and $x_{k}=0$ for all $k \in[1 \ldots K] \backslash J$.

Proof of Proposition 1.2 First, suppose $\beta \leq 1$ and let $\mu \in \Delta^{*}(X)$ be such that $\mu(\mathbf{x})>\frac{1}{2}$ for some $\mathbf{x} \in X$ and $\beta \leq 1$. Since $d^{\beta}$ is a concave function of $d$ if $\beta \leq 1$, the local minima 
of the function $\sum_{\mathbf{y} \in X}[d(\cdot, \mathbf{y})]^{\beta} \cdot \mu(\mathbf{y})$ must all be elements of $\operatorname{supp}(\mu)$, thus the global minimum is by assumption at $\mathbf{x}$.

Conversely, suppose $\beta>1$. Consider a triple $\mathbf{x}, \mathbf{y}, \mathbf{z} \in X$ such that $\mathbf{y}$ is between $\mathbf{x}$ and $\mathbf{z}$, and assume without loss of generality that $\mathbf{x} \asymp \mathbf{y}$ and $\mathbf{y} \asymp \mathbf{z}$. Let $A:=d(\mathbf{x}, \mathbf{y})$ and $B:=d(\mathbf{y}, \mathbf{z})$; thus, $A+B=d(\mathbf{x}, \mathbf{z})$. Let $\mu \in \Delta^{*}(X)$ be such that $\mu(\mathbf{x})=\frac{1}{2}+\varepsilon$ and $\mu(\mathbf{z})=\frac{1}{2}-\varepsilon$, for some $\varepsilon>0$. Thus, PM requires $F_{\beta}(\mu)=\{\mathbf{x}\}$.

However, the objective function $\sum_{\mathbf{r} \in X}[d(\cdot, \mathbf{r})]^{\beta} \cdot \mu(\mathbf{r})$ has the value $\left(\frac{1}{2}-\varepsilon\right) \cdot(A+B)^{\beta}$ at $\mathbf{x}$, whereas at $\mathbf{y}$, it has the value

$$
\left(\frac{1}{2}+\varepsilon\right) A^{\beta}+\left(\frac{1}{2}-\varepsilon\right) B^{\beta}<\left(\frac{1}{2}+\varepsilon\right)\left(A^{\beta}+B^{\beta}\right) .
$$

For any $\beta>1$, we have $\left(A^{\beta}+B^{\beta}\right)<(A+B)^{\beta}$. Thus, if $\varepsilon$ is sufficiently small, we have $\left(\frac{1}{2}+\varepsilon\right)\left(A^{\beta}+B^{\beta}\right)<\left(\frac{1}{2}-\varepsilon\right) \cdot(A+B)^{\beta}$; thus the minimum value does not occur at $\mathbf{x}$, so $F_{\beta}(\mu) \neq\{\mathbf{x}\}$, contradicting PM.

Proof of Proposition 1.3. It is straightforward to verify that $F_{1}$ (the median rule) is Condorcet consistent. We will prove the converse by contrapositive. If $\beta>1$, then Proposition 1.2 implies that $F_{\beta}$ violates PM; hence it cannot be CC. So, suppose $\beta<1$. Again, consider a triple $\mathbf{x}, \mathbf{y}, \mathbf{z} \in X$ such that $\mathbf{y}$ is between $\mathbf{x}$ and $\mathbf{z}$, and assume without loss of generality that $\mathbf{x} \asymp \mathbf{y}$ and $\mathbf{y} \asymp \mathbf{z}$. Let $A:=d(\mathbf{x}, \mathbf{y})$ and $B:=d(\mathbf{y}, \mathbf{z})$; thus, $A+B=d(\mathbf{x}, \mathbf{z})$. Let $C:=A^{\beta}+B^{\beta}-(A+B)^{\beta}$; then $C>0$ because $\beta<1$.

Let $\mu \in \Delta^{*}(X)$ be such that $\mu(\mathbf{x})=\mu(\mathbf{z})=\frac{1}{2}-\varepsilon$, while $\mu(\mathbf{y})=2 \varepsilon$, for some $\varepsilon>0$. It is easily checked that $\operatorname{Maj}(\mu)=\mathbf{y}$. Thus, $\mathrm{CC}$ requires $F_{\beta}(\mu)=\{\mathbf{y}\}$.

However, if we define $\phi(\mathbf{s}):=\sum_{\mathbf{r} \in X}[d(\mathbf{s}, \mathbf{r})]^{\beta} \cdot \mu(\mathbf{r})$ for all $\mathbf{s} \in X$, then $\phi(\mathbf{x})=\mu(\mathbf{y}) A^{\beta}+$ $\mu(\mathbf{z})(A+B)^{\beta}$, whereas $\phi(\mathbf{y})=\mu(\mathbf{x}) A^{\beta}+\mu(\mathbf{z}) B^{\beta}=\mu(\mathbf{z})\left(A^{\beta}+B^{\beta}\right)$. Thus,

$$
\begin{aligned}
\phi(\mathbf{y})-\phi(\mathbf{x}) & =\mu(\mathbf{z})\left(A^{\beta}+B^{\beta}\right)-\mu(\mathbf{y}) A^{\beta}-\mu(\mathbf{z})(A+B)^{\beta} \\
& =\mu(\mathbf{z}) C-\mu(\mathbf{y}) A^{\beta} \\
& =\left(\frac{1}{2}-\varepsilon\right) C-2 \varepsilon A^{\beta} .
\end{aligned}
$$

Recall $C>0$. Thus, if $\varepsilon$ is small enough, then $\phi(\mathbf{y})-\phi(\mathbf{x})>0$, which means $\phi(\mathbf{y})>\phi(\mathbf{x})$, which means $\mathbf{y} \notin F_{\beta}(\mu)$, contradicting CC.

Proof of Lemma 1.4. For any $\mu \in \Delta^{*}(X)$ and any $k \in[1 \ldots K]$, observe that

$$
\begin{aligned}
\left(\mu_{k}\left(x_{k}\right)>\mu_{k}\left(y_{k}\right)\right) & \Leftrightarrow\left(x_{k}=\operatorname{Maj}_{k}(\mu) \neq y_{k}\right) \Longrightarrow(k \in \mathcal{M}(\mathbf{x}, \mu)) \\
\text { while }\left(\mu_{k}\left(x_{k}\right)=\mu_{k}\left(y_{k}\right)\right) & \Leftrightarrow\left(x_{k}=y_{k}\right) \Longrightarrow(k \in \mathcal{M}(\mathbf{x}, \mu) \Leftrightarrow k \in \mathcal{M}(\mathbf{y}, \mu)),
\end{aligned}
$$

Thus, $\mu_{k}\left(x_{k}\right) \geq \mu_{k}\left(y_{k}\right)$ for all $k \in[1 \ldots K]$, if and only if either (A1) or (A2) applies for each $k \in[1 \ldots K]$, if and only if $\mathcal{M}(\mathbf{x}, \mu) \supseteq \mathcal{M}(\mathbf{y}, \mu)$. 
Proof of Lemma 1.5 (a) is immediate from the definition of Condorcet admissibility, as is the first part of (b). Now suppose $\operatorname{Maj}(\mu) \notin X$; it remains only to show that $\mid$ Cond $(X, \mu) \mid \geq 3$. To see this, note that $\operatorname{Maj}(\mu) \notin X$ only if there is some $X$-critical fragment $\mathbf{w}$ which is activated by $\mu$. This fragment $\mathbf{w}$ must have order 3 or more (if $|\mathbf{w}|=2$, then $\mathbf{w}$ could not be activated by $\mu$ : if each coordinate received majority support, then a nonzero proportion of voters would endorse both coordinates of $\mathbf{w}$, which is impossible because $\mathbf{w}$ is forbidden).

Now, fix some coordinate $j \in \operatorname{supp}(\mathbf{w})$, and let $\mathbf{w}^{j}$ be the fragment obtained by deleting coordinate $j$ from $\mathbf{w}$. Let $X_{j}:=\left\{\mathbf{x} \in X ; \mathbf{w}^{j} \sqsubset \mathbf{x}\right\}$; then $X_{j}$ is nonempty, because $\mathbf{w}^{j}$ is not forbidden, because $\mathbf{x}$ is critical. Let $\mathbf{x}$ be an element of $X_{j}$ such that there exists no $\mathbf{y} \in X_{j}$ with $\mathcal{M}(\mathbf{x}, \mu) \subsetneq \mathcal{M}(\mathbf{y}, \mu)$.

Claim 1: $\quad \mathbf{x} \in \operatorname{Cond}(X, \mu)$.

Proof: Suppose there was some $\mathbf{y} \in X$ with $\mathcal{M}(\mathbf{x}, \mu) \subsetneq \mathcal{M}(\mathbf{y}, \mu)$. Then we must have $\mathbf{y} \notin X_{j}$, which means $y_{j}=w_{j}$. But $\operatorname{supp}(\mathbf{w}) \backslash\{j\} \subseteq \mathcal{M}(\mathbf{x}, \mu) \subset \mathcal{M}(\mathbf{y}, \mu)$, so $\mathbf{w}^{j} \sqsubset \mathbf{y}$. Thus, $\mathbf{w} \sqsubset \mathbf{y}$. But $\mathbf{w}$ is forbidden, so this is impossible for any $\mathbf{y} \in X$. $\diamond$ claim 1

Thus, we can obtain an element of Cond $(X, \mu)$ by contradicting the majority will in any single coordinate of $\mathbf{w}$; thus, there are at least as many different elements of Cond $(X, \mu)$ as there are coordinates in $\mathbf{w}$ - hence $\mid$ Cond $(X, \mu)|\geq| \mathbf{w} \mid \geq 3$.

Proof of Proposition 1.7 To show that any weighted additive majority rule $F_{\phi, \lambda}$ is Condorcet admissible, take two views $\mathbf{x}$ and $\mathbf{y}$ such that $\mathbf{x}$ agrees with the majority view on a strictly larger set of issues than $\mathbf{y}$; say $\mathbf{x}$ agrees with $\operatorname{Maj}(\mu)$ on $K_{\mathbf{x}} \subseteq[1 \ldots K]$, and $\mathbf{y}$ agrees with $\operatorname{Maj}(\mu)$ on $K_{\mathbf{y}} \subseteq[1 \ldots K]$, with $K_{\mathbf{x}} \supsetneqq K_{\mathbf{y}}$. If $k \in K_{\mathbf{y}}$, or if $k \notin K_{\mathbf{x}}$, then $\mu_{k}\left(x_{k}\right)=\mu_{k}\left(y_{k}\right)$; on the other hand, if $k \in K_{\mathbf{x}} \backslash K_{\mathbf{y}}$, then

$$
\mu_{k}\left(x_{k}\right)-\frac{1}{2}>0>\mu_{k}\left(y_{k}\right)-\frac{1}{2},
$$

hence $\phi\left[\mu_{k}\left(x_{k}\right)-\frac{1}{2}\right]>\phi\left[\mu_{k}\left(y_{k}\right)-\frac{1}{2}\right]$ by our assumptions on $\phi$. This shows that $\mathbf{y}$ does not maximize the sum on the right hand side of (4), and hence that $\mathbf{y}$ is not chosen by $F_{\phi, \lambda}$.

Conversely, let a gain function $\phi$ and a view $\mathbf{x} \in \operatorname{Cond}(X, \mu)$ be given. Suppose that $\mathbf{x}$ agrees with the majority view in the set $K_{\mathbf{x}} \subseteq[1 \ldots K]$ of issues, and let $m:=\left|K_{\mathbf{x}}\right|$ (note that $m>0$ ). If $m=K$, then $\mathbf{x}$ agrees with the majority view in all issues and is the unique Condorcet admissible view by Lemma 1.5. Thus, assume that $m<K$. For $\varepsilon>0$, define $\lambda_{k}(\varepsilon)=\frac{1}{m}-\varepsilon$ if $k \in K_{\mathbf{x}}$ and $\lambda_{k}(\varepsilon)=\frac{m \varepsilon}{K-m}$ if $k \notin K_{\mathbf{x}}$. If $\varepsilon$ is sufficiently small, we obtain $0<\lambda_{k}(\varepsilon)<1$ for all $k$. Moreover, for sufficiently small $\varepsilon$ the view $\mathbf{x}$ is the unique maximizer of the right hand side of (4). Indeed, any other view $\mathbf{y} \in \operatorname{Cond}(X, \mu)$ disagrees with the majority view in least one issue $k \in K_{\mathbf{x}}$. Since, for all $k \notin K_{\mathbf{x}}, \lambda_{k}(\varepsilon)$ tends to zero, and $\phi\left[\mu_{k}\left(x_{k}\right)-\frac{1}{2}\right]>0$ for all $k \in K_{\mathbf{x}}$, this shows that $\mathbf{y}$ is not chosen by $F_{\phi, \lambda(\varepsilon)}$ for sufficiently small $\varepsilon$. 
Proof of Proposition 1.8. Let $\mathbf{z}:=\operatorname{Maj}(\mu)$.

(a) Let $\gamma$ be a path through $[1 \ldots K]$, and let $\mathbf{x}:=F^{\gamma}(\mu)$. We must show that $\mathbf{x} \in$ Cond $(X, \mu)$. Let $\mathbf{y} \in X$, and suppose $\mathbf{y}$ is between $\mathbf{x}$ and $\mathbf{z}$.

Claim 1: For all $t \in[1 \ldots K]$, we have $x_{\gamma(t)}=y_{\gamma(t)}$.

Proof: (by induction on $t$ ) First, $x_{\gamma(1)}=z_{\gamma(1)}$ (by definition of $F^{\gamma}$ ). Thus, $y_{\gamma(1)}=x_{\gamma(1)}$ also (because $\mathbf{y}$ is between $\mathbf{x}$ and $\mathbf{z}$ ).

Now, let $J:=\{\gamma(1), \ldots, \gamma(t-1)\}$, and suppose inductively that $\mathbf{x}_{J}=\mathbf{y}_{J}$; we will show that $x_{\gamma(t)}=y_{\gamma(t)}$. If $x_{\gamma(t)}=z_{\gamma(t)}$, then $y_{\gamma(t)}=x_{\gamma(t)}$ (because $\mathbf{y}$ is between $\mathbf{x}$ and $\mathbf{z})$. If $x_{\gamma(t)} \neq z_{\gamma(t)}$, then this must be because $z_{\gamma(t)}$ is $X$-inconsistent with $\mathbf{x}_{J}$. But then $z_{\gamma(t)}$ is also $X$-inconsistent with $\mathbf{y}_{J}$ (by induction), so we must also have $y_{\gamma(t)} \neq z_{\gamma(t)}$ (because $\mathbf{y} \in X$ ). Thus, $y_{\gamma(t)}=x_{\gamma(t)}$. $\quad$ claim 1

Thus, if $\mathbf{y}$ is between $\mathbf{z}$ and $\mathbf{x}$, then Claim 1 implies that $\mathbf{y}$ is $\mathbf{x}$. Thus, $\mathbf{x} \asymp \mathbf{z}$; hence $\mathbf{x} \in \operatorname{Cond}(X, \mu)$, as desired.

(b) Let $\mathbf{x} \in$ Cond $(X, \mu)$; we must find a path $\gamma$ such that $F^{\gamma}(\mu)=\mathbf{x}$. Let $J:=$ $|\mathcal{M}(\mathbf{x}, \mu)|$, and let $\gamma:[1 \ldots K] \longrightarrow[1 \ldots K]$ be a path such that $\gamma[1 \ldots J]=\mathcal{M}(\mathbf{x}, \mu)$. Thus, $x_{\gamma(j)}=z_{\gamma(j)}$ for all $j \in[1 \ldots J]$, while $x_{\gamma(j)} \neq z_{\gamma(j)}$ for all $j \in[J+1 \ldots K]$. Let $\mathbf{y}:=F^{\gamma}(\mu)$.

Claim 2: $\quad$ For all $t \in[1 \ldots J], y_{\gamma(t)}=x_{\gamma(t)}$.

Proof: (by induction on $t$ ) First, $y_{\gamma(1)}=z_{\gamma(1)}$ by definition of $F^{\gamma}$; hence $y_{\gamma(1)}=x_{\gamma(1)}$.

Let $t \in[1 \ldots J]$, let $I:=\{\gamma(1), \ldots, \gamma(t-1)\}$ and suppose inductively that $\mathbf{y}_{I}=\mathbf{x}_{I}$. Now, $x_{\gamma(t)}$ is $X$-consistent with $\mathbf{x}_{I}$ (because $\mathbf{x} \in X$ ); hence $z_{\gamma(t)}$ is $X$-consistent with $\mathbf{y}_{I}$ (because $\mathbf{y}_{I}=\mathbf{x}_{I}$ by induction hypothesis, while $z_{\gamma(t)}=x_{\gamma(t)}$ by definition of $\gamma$ and $J)$. Thus, $y_{\gamma(t)}=z_{\gamma(t)}$; hence $y_{\gamma(t)}=x_{\gamma(t)}$.

$\diamond$ Claim 2

Claims 2 implies $\mathbf{y}_{J}=\mathbf{x}_{J}$; hence $\mathbf{y}$ is between $\mathbf{x}$ and $\mathbf{z}$ (by definition of $J$ ). But $\mathbf{y} \in X$ and $\mathbf{x} \asymp \mathbf{z}$; thus we must have $\mathbf{y}=\mathbf{x}$-in other words, $F^{\gamma}(\mu)=\mathbf{x}$, as desired.

Proof of Proposition 1.9. "[i] $\Longleftrightarrow$ [ii]" follows immediately from Lemma 1.5 and Propo-

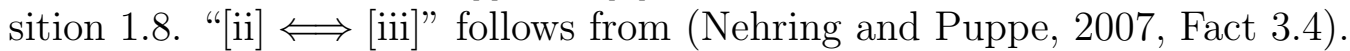

The proof of Theorem 2.1 is somewhat involved, so it is deferred until the end of the Appendix.

Proof of Proposition 3.1. (a) " $\subseteq$ " (by contrapositive) Let $\mathrm{x} \in X_{N}^{\mathrm{pr}}$ represent the ordering $\left(a_{1} \stackrel{\mathbf{x}}{\prec} a_{2} \stackrel{\mathbf{x}}{\prec} a_{3} \stackrel{\mathbf{x}}{\prec} \ldots \stackrel{\text { }}{\prec} a_{N}\right)$, and suppose $\stackrel{\mathbf{x}}{\prec}$ is not a directed Hamiltonian chain in $\stackrel{\mu}{\prec}$. Then there exists $n \in[1 \ldots N)$ such that $a_{n} \iota^{\stackrel{\mu}{\zeta}} a_{n+1}$. Define the ordering $\stackrel{\mathrm{y}}{\prec}$ by switching the positions of $a_{n}$ and $a_{n+1}$ in $\stackrel{\mathrm{x}}{\prec}$. That is: $a_{1} \stackrel{\mathrm{y}}{\prec} a_{2} \stackrel{\mathrm{y}}{\prec} \ldots \stackrel{\mathrm{y}}{\prec} a_{n-2} \stackrel{\mathrm{y}}{\prec} a_{n-1} \stackrel{\mathrm{y}}{\prec} a_{n+1} \stackrel{\mathrm{y}}{\prec} a_{n}$ $\stackrel{\mathrm{y}}{\prec} a_{n+2} \stackrel{\mathrm{y}}{\prec} a_{n+3} \stackrel{\mathrm{y}}{\prec} \ldots \stackrel{\mathrm{y}}{\prec} a_{N}$. Observe that $\stackrel{\mathrm{y}}{\prec}$ agrees with $\stackrel{\mathrm{x}}{\prec}$ in every pairwise ordering except the ordering of $\left\{a_{n}, a_{n+1}\right\}$. Thus, $\mathcal{M}(\mathbf{y}, \mu)=\mathcal{M}(\mathbf{x}, \mu) \cup\left\{\left(a_{n} \prec a_{n+1}\right)\right\}$. Thus, $\mathcal{M}(\mathbf{x}, \mu)$ is not maximal, so $\mathbf{x} \notin \operatorname{Cond}\left(X_{N}^{\mathrm{pr}}, \mu\right)$. 
“?" Let $\left(a_{1} \stackrel{\mu}{\prec} a_{2} \stackrel{\mu}{\prec} \ldots \stackrel{\mu}{\prec} a_{N}\right)$ be any directed Hamiltonian chain in $\stackrel{\mu}{\prec}$. Let $\mathbf{x} \in X_{N}^{\text {pr }}$ be the transitive closure of this Hamiltonian chain (so that $a_{1} \stackrel{\mathrm{x}}{\prec} a_{2} \stackrel{\mathbf{x}}{\prec} \cdots \stackrel{\mathrm{x}}{\prec} a_{N}$ ). Then $\mathcal{M}(\mathbf{x}, \mu) \supseteq\left\{\left(a_{1} \prec a_{2}\right),\left(a_{2} \prec a_{3}\right), \ldots,\left(a_{N-1} \prec a_{N}\right)\right\}$. Furthermore, for any $\mathbf{y} \in X_{N}^{\mathrm{pr}}$, if $\mathcal{M}(\mathbf{y}, \mu) \supseteq\left\{\left(a_{1} \prec a_{2}\right),\left(a_{2} \prec a_{3}\right), \ldots,\left(a_{N-1} \prec a_{N}\right)\right\}$, then clearly $\mathbf{y}=\mathbf{x}$. Thus, $\mathcal{M}(\mathbf{x}, \mu)$ is maximal, so $\mathbf{x} \in \operatorname{Cond}\left(X_{N}^{\mathrm{pr}}, \mu\right)$.

(b) " $\Rightarrow$ " (by contrapositive) Suppose there is some $\mathbf{x} \in \operatorname{Cond}\left(X_{N}^{\mathrm{pr}}, \mu\right)$ with $a \stackrel{\mathrm{x}}{\prec} b$. Relabel $[1 \ldots N]=\left\{a_{1}, \ldots, a_{N}\right\}$ such that $a_{1} \stackrel{x}{\prec} a_{2} \stackrel{x}{\prec} \ldots \stackrel{\mathrm{x}}{\prec} a_{N}$. Then $a=a_{k}$ and $b=a_{l}$ for some $k<l$. By part (a), the nearest-neighbour orderings in $\stackrel{\times}{\prec}$ agree with the

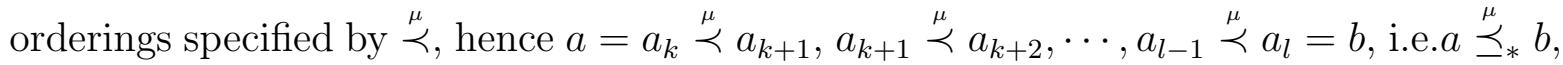
which means $a \ddot{\nsucc}_{*} b$.

Thus, contrapositively, if $a \stackrel{\mu}{\succ}_{*} b$, then we must have $a \stackrel{\mathrm{x}}{\succ} b$ for all $\mathbf{x} \in \operatorname{Cond}\left(X_{N}^{\mathrm{pr}}, \mu\right)$.

" that $a \neq b$. We will construct $\mathbf{x} \in \operatorname{Cond}\left(X_{N}^{\mathrm{pr}}, \mu\right)$ with $a \stackrel{\mathrm{x}}{\prec} b$. Let $A:=\left\{a_{k}, a_{k+1}, \cdots, a_{l}\right\}$ be a minimal set such that $a_{k}=a, a_{l}=b$ and $a_{k} \stackrel{\mu}{\prec} a_{k+1}, a_{k+1} \stackrel{\mu}{\prec} a_{k+2}, \cdots, a_{l-1} \stackrel{\mu}{\prec} a_{l}$. Consider any path $\gamma$ that decides the issues $\left(a_{k}, a_{k+1}\right),\left(a_{k+1}, a_{k+2}\right), \ldots,\left(a_{l-1}, a_{l}\right)$ first, and let $\mathbf{x}:=F^{\gamma}(\mu)$. By minimality of $A$, there are no transitivity constraints among these first $l-k$ decisions, thus they all agree with the majority view, i.e. $a=a_{k} \stackrel{\mathrm{x}}{\prec}$ $a_{k+1} \stackrel{\mathrm{x}}{\prec} \cdots \stackrel{\mathrm{x}}{\prec} a_{l}=b$. Hence, $a \stackrel{\mathrm{x}}{\prec} b$ by transitivity.

Thus, contrapositively, if $a \stackrel{\mathrm{x}}{\succ} b$ for all $\mathbf{x} \in$ Cond $\left(X_{N}^{\mathrm{pr}}, \mu\right)$, then we must have $a \stackrel{\mu}{\succ}{ }_{*} b$.

(c) Part (b) implies that $\mu$ is globally indeterminate if and only if, for all $a, b \in[1 \ldots N]$, neither $a \stackrel{\mu}{\succ}_{*} b$ nor $b \stackrel{\iota}{\succ}_{*} a$. This is the case if and only if, for all $a, b \in[1 \ldots N]$, we have both $b \succeq_{*}^{\mu} a$ and $a \bigsqcup_{*}^{\mu} b$-i.e. there is a directed path from $a$ to $b$ in the majority tournament, and also a directed path from $b$ to $a$ in the majority tournament. This statement, in turn, is true if and only if all elements of $[1 \ldots N]$ are part of the same cycle -i.e. the top cycle of $\mu$ encompasses all of $[1 \ldots N]$.

Proof of Proposition 3.4. (a) " there is some $\mathbf{y} \in X_{N}^{\text {eq }} \backslash\{\mathbf{x}\}$ with $\mathbf{y} R(\mu) \mathbf{x}$; thus, Lemma 1.4 implies that $\mathcal{M}(\mathbf{x}, \mu) \subsetneq$ $\mathcal{M}(\mathbf{y}, \mu)$. Thus, there exist some $a, b \in[1 \ldots N]$ such that $(a, b) \in \mathcal{M}(\mathbf{y}, \mu) \backslash \mathcal{M}(\mathbf{x}, \mu)$; in other words, $x_{(a, b)} \neq y_{(a, b)}=x_{(a, b)}^{\mu}$.

First suppose $x_{(a, b)}^{\mu}=y_{(a, b)}=0$ whereas $x_{(a, b)}=1$. Thus, $a \stackrel{\mathrm{x}}{\sim} b$, but $a \stackrel{\mathrm{y}}{\sim} b$. Now, let $C \subseteq[1 \ldots N]$ be the $\stackrel{\mathbf{x}}{\sim}$-equivalence class containing $a$ and $b$; we show that $C$ is not connected in the graph defined by $\mathbf{x}^{\mu}$. To see this, let $a=c_{0}, c_{1}, c_{2}, \ldots, c_{L}=b$ be any putative path in $C$ connecting $a$ with $b$. Then there must exist some $\ell \in[1 \ldots L]$ such that $c_{\ell-1} \stackrel{\mathrm{y}}{\sim} c_{\ell}$ (because $a \stackrel{\mathrm{y}}{\sim} b$ ). But $c_{\ell-1} \stackrel{\mathrm{x}}{\sim} c_{\ell}$ (because $\left.c_{0}, c_{1}, \ldots, c_{L} \in C\right)$. In other words, $y_{\left(c_{\ell-1}, c_{\ell}\right)}=0$ whereas $x_{\left(c_{\ell-1}, c_{\ell}\right)}=1$. Thus, we must have $x_{\left(c_{\ell-1}, c_{\ell}\right)}^{\mu}=0$ (otherwise $\mathcal{M}(\mathbf{x}, \mu) \nsubseteq \mathcal{M}(\mathbf{y}, \mu))$. Thus, $\left(c_{0}, c_{1}, \ldots, c_{L}\right)$ is not a path in the graph defined by $\mathbf{x}^{\mu}$. 
Thus, $C$ is not connected as a subset of the $\mathbf{x}^{\mu}$-graph, hence $\stackrel{\mathbf{x}}{\sim} \nsubseteq \operatorname{tr}\left(\mathbf{x}^{\mu}\right)$, i.e. $\mathbf{x}$ violates condition [i].

Suppose instead that $\mathbf{x}$ satisfies condition [i], but that there is some $a, b \in[1 \ldots N]$ such that $x_{(a, b)}^{\mu}=y_{(a, b)}=1$ whereas $x_{(a, b)}=0$. Thus, $a \stackrel{\mathbf{y}}{\sim} b$, but $a \stackrel{\mathbf{x}}{\sim} b$. Thus, $a$ and $b$ lie in different $\stackrel{\mathrm{x}}{\sim}$-equivalence classes; call them $A$ and $B$. Let $C$ be the $\stackrel{\mathbf{y}}{\sim}$-equivalence class which contains both $a$ and $b$.

Claim 1: $A \cup B \subseteq C$.

Proof: Since $\mathbf{x}$ satisfies condition [i], the $\stackrel{\mathbf{x}}{\sim}$-equivalence class $A$ lies in an $\mathbf{x}^{\mu}$-connected component. Thus, for any $c \in A$, there exists a path $a=c_{0}, c_{1}, \ldots, c_{L}=c$ in $A$ such that $x_{\left(c_{\ell-1}, c_{\ell}\right)}^{\mu}=1$ for all $\ell \in[1 \ldots L]$. And of course, $x_{\left(c_{\ell-1}, c_{\ell}\right)}=1$ (because $c_{\ell-1} \stackrel{\mathbf{x}}{\sim} c_{\ell}$ because they are both in $A$ ). Thus, we must have $y_{\left(c_{\ell-1}, c_{\ell}\right)}=1$ (otherwise $\mathcal{M}(\mathbf{x}, \mu) \nsubseteq \mathcal{M}(\mathbf{y}, \mu))$. Thus $c_{\ell-1} \stackrel{\mathbf{y}}{\sim} c_{\ell}$ for all $\ell \in[1 \ldots L]$. Thus, $a \stackrel{\mathbf{y}}{\sim} c$ by transitivity. This holds for all $c \in A$, so $A \subseteq C$. By an identical argument, $B \subseteq C . \quad \diamond$ claim 1

Now, for any $a^{\prime} \in A$ and $b^{\prime} \in B$, we have $x_{\left(a^{\prime}, b^{\prime}\right)}=0$ (because $a^{\prime} \stackrel{\mathbf{x}}{\mathcal{\psi}} b^{\prime}$ ) but $y_{\left(a^{\prime}, b^{\prime}\right)}=1$ (because $a^{\prime} \stackrel{\text { y }}{\sim} b^{\prime}$ because $a^{\prime} \in A \subset C$ and $b^{\prime} \in B \subset C$ by Claim 1). Thus, we must have $x_{\left(a^{\prime}, b^{\prime}\right)}^{\mu}=1$ (otherwise $\left.\mathcal{M}(\mathbf{x}, \mu) \nsubseteq \mathcal{M}(\mathbf{y}, \mu)\right)$. Thus, condition [ii] is violated.

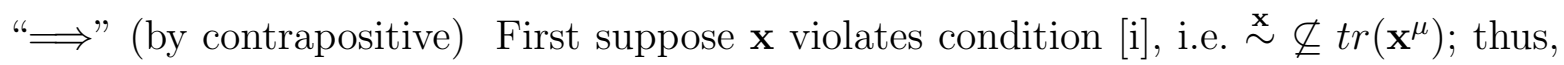
there is some equivalence class $B \subseteq[1 \ldots N]$ of $\stackrel{\mathrm{x}}{\sim}$ which is not connected in the graph defined by $\mathbf{x}^{\mu}$. Thus, $B=C \sqcup D$, where for all $c \in C$ and $d \in D$, we have $x_{(c, d)}^{\mu}=0$ (whereas $\left.x_{(c, d)}=1\right)$. Define $\mathbf{y} \in\{0,1\}^{K}$ to be identical to $\mathbf{x}$, except that $y_{(c, d)}:=0$ for all $c \in C$ and $d \in D$. Then it is easy to verify that $\mathbf{y} \in X_{N}^{\text {eq }}$, and $\mathcal{M}(\mathbf{y}, \mu)=\mathcal{M}(\mathbf{x}, \mu) \sqcup\{(c, d)$; $c \in C$ and $d \in D\}$; thus, Lemma 1.4 implies that $\mathbf{y} R(\mu) \mathbf{x}$, so $\mathbf{x} \notin$ Cond $\left(X_{N}^{\mathrm{eq}}, \mu\right)$.

Now suppose $\mathbf{x}$ violates condition [ii]. Thus, there exist distinct $\stackrel{\mathbf{x}}{\sim}$-equivalence classes $B, C \subset[1 \ldots N]$ such that, for all $b \in B$ and $c \in C$, we have $x_{(b, c)}^{\mu}=1$ (whereas $x_{(b, c)}=0$ ). Define $\mathbf{y} \in\{0,1\}^{K}$ to be identical to $\mathbf{x}$, except that $y_{(b, c)}:=1$ for all $b \in B$ and $c \in C$. Then it is easy to verify that $\mathbf{y} \in X_{N}^{\mathrm{eq}}$, and $\mathcal{M}(\mathbf{y}, \mu)=\mathcal{M}(\mathbf{x}, \mu) \sqcup\{(b, c) ; \quad b \in B$ and $c \in C\}$; thus, Lemma 1.4 implies that $\mathbf{y} R(\mu) \mathbf{x}$, so $\mathbf{x} \notin \operatorname{Cond}\left(X_{N}^{\mathrm{eq}}, \mu\right)$.

(b) The second part follows from part (a) in a straightforward manner.

(c) Part (b) implies that $\mu$ is globally indeterminate if and only if, for all $a, b \in[1 \ldots N]$, either

(1) $\mathbf{x}_{(a, b)}^{\mu}=0$ but $\operatorname{tr}(\mathbf{x})_{(a, b)}^{\mu}=1$, or

(2) $\mathbf{x}_{(a, b)}^{\mu}=1$ but $\mathbf{x}_{(c, d)}^{\mu}=0$ for some vertices $c$ and $d$ in the same connected component as $a$ and $b$.

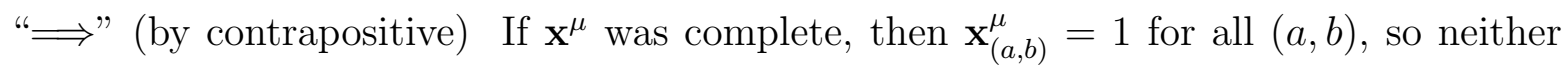
Case (1) nor Case (2) could ever occur. Thus, $\mathbf{x}^{\mu}$ cannot be complete if there is to be any indeterminacy. 
On the other hand if $\mathbf{x}^{\mu}$ was disconnected, then for any $a, b \in[1 \ldots N]$ in different connected components, we would have $\mathbf{x}_{(a, b)}^{\mu}=\operatorname{tr}(\mathbf{x})_{(a, b)}^{\mu}=0$, so neither Case (1) nor Case (2) could ever occur for these points. It follows that for either Case (1) or Case (2) to ever occur, $\mathrm{x}^{\mu}$ must be connected, but not complete.

"£" Suppose $\mathbf{x}^{\mu}$ was connected but not complete. Then there is some $c, d \in[1 \ldots N]$ such that $\mathbf{x}_{(c, d)}^{\mu}=0$. Furthermore, $c$ and $d$ belong to the same connected component as every other vertex in $[1 \ldots N]$. Thus, for any $a, b \in[1 \ldots N]$ such that $\mathbf{x}_{(a, b)}^{\mu}=1$, we have an instance of Case (2), so the edge $(a, b)$ is indeterminate. On the other hand, for any $a, b \in[1 \ldots N]$ such that $\mathbf{x}_{(a, b)}^{\mu}=0$, we must still have $\operatorname{tr}(\mathbf{x})_{(a, b)}^{\mu}=1$ (because $\mathbf{x}^{\mu}$ is connected), so we get an instance of Case (1), so again the edge $(a, b)$ is indeterminate. In this way, we see that all edges are indeterminate, so $\mu$ is globally indeterminate.

Proof of Proposition 3.5. Observe that $\mathbf{1}_{M}=\operatorname{Maj}(\mu)$.

(a) If $I \leq|M| \leq J$, then $\mathbf{1}_{M} \in X_{I, J ; K}^{\text {com }}$, so Cond $\left(X_{I, J ; K}^{\mathrm{com}}, \mu\right)=\left\{\mathbf{1}_{M}\right\}$.

(b) If $|M|<I$, then $\mathbf{1}_{M} \notin X_{I, J ; K}^{\text {com }}$, and for any $G, H \subseteq[1 \ldots K]$ if $\mathbf{1}_{G}, \mathbf{1}_{H} \in X_{I, J ; K}^{\text {com }}$, then we have $\mathbf{1}_{G} M(\mu) \mathbf{1}_{H}$ if and only if $H \cap M \subseteq G \cap M$ while $G \backslash M \subseteq H \backslash M$. Thus, $\mathbf{1}_{H} \in$ Cond $\left(X_{I, J ; K}^{\mathrm{com}}, \mu\right)$ if and only if $M \subset H$ and there is no $\mathbf{1}_{G} \in X_{I, J ; K}^{\mathrm{com}}$ with $M \subset G \subset H$-in other words, if $M \subset H$ and $|H|=I$.

The proof of (c) is similar to (b).

The following lemma will be useful in the proof of Proposition 3.6.

Lemma A.1 Consider the committee selection problem $X_{I . J ; K}^{\text {com }}$. For all $I \leq J$, we have $\operatorname{Crit}\left(X_{I . J ; K}^{\mathrm{com}}\right) \subseteq\{\mathbf{0}, \mathbf{1}\}$. Moreover, if $I>0$ then $\mathbf{0} \in \operatorname{Crit}\left(X_{I, J ; K}^{\mathrm{com}}\right)$, and if $J<K$ then $1 \in \operatorname{Crit}\left(X_{I, J ; K}^{\mathrm{com}}\right)$.

Proof: Evidently, the critical fragments of $X_{I, J ; K}^{\text {com }}$ are given as follows: if $I>0$, then all fragments of exactly $K-I+1$ zeros are critical; moreover, if $J<K$, then all fragments of exactly $J+1$ ones are critical. No other fragments are critical. This implies at once that $\mathbf{0} \in \operatorname{Crit}\left(X_{I, J ; K}^{\mathrm{com}}\right)$ if $I>0$, and $\mathbf{1} \in \operatorname{Crit}\left(X_{I, J ; K}^{\mathrm{com}}\right)$ if $J<K$. Moreover, $I \leq J$, so $(K-I+1)+(J+1)>K$, so no element $\mathbf{x} \in\{0,1\}^{K}$ different from $\mathbf{0}$ and $\mathbf{1}$ can be critical for $X_{I, J ; K}^{\mathrm{com}}$.

Proof of Proposition 3.6. By Lemma A.1, $\operatorname{Crit}\left(X_{I . J ; K}^{\mathrm{com}}\right)=\{\mathbf{0}, \mathbf{1}\}$. First, assume that $K / 2<J$. For each $l \in[1 \ldots K]$, denote by $\mathbf{x}^{l}$ the element of $X_{I, J ; K}^{\text {com }}$ with $x_{k}^{l}=1$ for $k=l, l+1, \ldots, l+J(\bmod K)$, and $x_{k}=0$ otherwise. Evidently, for the profile $\mu$ that assigns weight $1 / K$ to each $\mathbf{x}^{l}, l \in[1 \ldots K]$, one obtains $\operatorname{Maj}(\mu)=1$. Next, assume that $I<K / 2$; by a completely symmetric argument, one shows that $\mathbf{0} \in \operatorname{Maj}\left(X_{I, J ; K}^{\text {com }}\right)$ in this case. Thus, in either case $X_{I, J ; K}^{\mathrm{com}}$ is issue-wise indeterminate by Theorem 2.2(b).

Now assume that $J \leq K / 2 \leq I$, which is only possible if $K$ is even and $I=J=K / 2$. As is easily verified, $\operatorname{Maj}\left(X_{\frac{K}{2}, \frac{K}{2} ; K}^{\mathrm{com}}\right) \cap\{\mathbf{0}, \mathbf{1}\}=\emptyset$ in this case. For instance, suppose 
that $\operatorname{Maj}(\mu)$ specifies a zero in each of the first $K-1$ coordinates, then $\mu$ must contain strictly more ones than zeros in coordinate $K$; hence $[\operatorname{Maj}(\mu)]_{K}=1$. Thus, $X_{\frac{K}{2}, \frac{K}{2} ; K}^{\text {com }}$ is not issue-wise indeterminate, again by Theorem $2.2(\mathrm{~b})$.

Notation A.2. Let $J \subset[1 \ldots K]$, and let $\mathbf{w} \in\{0,1\}^{J}$. We define $[\mathbf{w}]:=\left\{\mathbf{x} \in\{0,1\}^{K}\right.$; $\mathbf{w} \sqsubseteq \mathbf{x}\}$. Thus, $\mathbf{w}$ is $X$-forbidden if and only if $X \cap[\mathbf{w}]=\emptyset$. It is sometimes convenient to express $\mathbf{w}$ as an element of $\{0,1, *\}^{K}$, where we define $w_{k}=*$ for all $k \notin J$. For example, suppose $J=\{i, i+1, \ldots, j\}$ for some $i \leq j \leq K$; then we would write $\mathbf{w}=$ $(\underbrace{*, \ldots, *}_{i-1}, w_{i}, w_{i+1}, \ldots, w_{j}, \underbrace{*, \ldots, *}_{K-j})$.

Proof of Proposition 3.7. We must show that $\operatorname{Crit}(X)=\{\mathbf{0}\}$.

Claim 1: Let $\mathbf{w}$ be any $X$-critical fragment. Then $\mathbf{w}$ is all zeros.

Proof: (by contradiction) Suppose w was not all zeros. By reordering $[1 \ldots K]$ if necessary, suppose that

$$
\mathbf{w}=(\underbrace{1,1, \ldots, 1}_{N}, \underbrace{0, \ldots, 0}_{M}, *, \ldots, *) .
$$

Let $\mathbf{w}^{\prime}=(*, \overbrace{1, \ldots, 1}^{N-1}, \overbrace{0, \ldots, 0}^{M}, *, \ldots, *)$. Then $\mathbf{w}^{\prime}$ is not $X$-forbidden (because $\mathbf{w}$ is $X$-critical). Thus, there exists some $\mathbf{x}^{\prime} \in X$ such that $\mathbf{w}^{\prime} \sqsubset \mathbf{x}$. Define $\mathbf{x} \in\{0,1\}^{K}$ by $x_{1}:=1$ and $x_{k}:=x_{k}^{\prime}$ for all $k \geq 2$. Then $\mathbf{x} \geq \mathbf{x}^{\prime}$. Thus, $\mathbf{x} \in X$ because $\mathbf{x}^{\prime} \in X$ and $X$ is comprehensive. But $\mathbf{w} \sqsubset \mathbf{x}$, and $\mathbf{w}$ is $X$-forbidden. Contradiction. $\quad \diamond$ claim 1

Now, by nondegeneracy, for every $k \in[1 \ldots K]$ there is some critical fragment $\mathbf{w}_{k} \in W_{3}(X)$ such that $k \in \operatorname{supp}\left(\mathbf{w}_{k}\right)$. By Claim $1, \mathbf{w}_{k}$ is all zeros. Thus, the fragment $\left\{\mathbf{w}_{k}\right\}_{k=1}^{K}$ is a 'critical covering' of $\mathbf{0}$, so $\mathbf{0} \in \operatorname{Crit}(X)$.

Conversely if $\mathbf{x} \in \operatorname{Crit}(X)$, then we must have $\mathbf{x}=\mathbf{0}$, because Claim 1 says that the families 'covering' $\mathbf{x}$ are all zeros. Thus, $\operatorname{Crit}(X)=\{\mathbf{0}\}$.

Proof of Corollary 3.8. $\quad X_{I, K ; K}^{\mathrm{com}}$ is comprehensive, so we can apply Proposition 3.7.

“” (by contrapositive) Suppose $I \geq K / 2$. Let $\mu \in \Delta^{*}\left(X_{I, K ; K}^{\mathrm{com}}\right)$. Observe that

$$
\sum_{k=1}^{K} \mu_{k}(1)=\sum_{k=1}^{K} \sum_{\mathbf{x} \in X} \mu[\mathbf{x}] x_{k}=\sum_{\mathbf{x} \in X} \mu[\mathbf{x}] \sum_{k=1}^{K} x_{k} \geq \sum_{\mathbf{x} \in X} \mu[\mathbf{x}] I \geq \frac{K}{2} .
$$

Thus, there must exist some $k \in[1 \ldots K]$ such that $\mu_{k}(1)>\frac{1}{2}$, and hence $\operatorname{Maj}_{k}(\mu)=1$. Thus, $\mathbf{0} \notin \operatorname{Maj}\left(X_{I, K ; K}^{\text {com }}\right)$; thus, Proposition 3.7 says $X_{I, K ; K}^{\text {com }}$ is not globally indeterminate. "£" Suppose $I<K / 2$. Let $\mu$ be a measure which is uniformly distributed on the set $X^{\prime}:=\left\{\mathbf{x} \in\{0,1\}^{K} ;|\mathbf{x}|=I\right\}$ (a subset of $X_{I, K ; K}^{\text {com }}$ ). Then for all $k \in[1 \ldots K]$, $\mu_{k}(1)=I / K<\frac{1}{2}$, so $\operatorname{Maj}_{k}(\mu)=0$. Thus, $\mathbf{0} \in \operatorname{Maj}\left(X_{I, K ; K}^{\text {com }}\right)$; thus, Proposition 3.7 says $X_{I, K ; K}^{\text {com }}$ is globally indeterminate. 
To prove Proposition 3.9, we need a lemma and some further notation. Recall (footnote 10) that the Slater rule minimizes the Hamming distance to $\operatorname{Maj}(\mu)$. The next lemma clarifies what this means in the discrete cube $[0 \ldots M]^{Q}$. For any $\mathbf{x}, \mathbf{y} \in[0 \ldots M]^{Q}$, we define

$$
d_{1}(\mathbf{x}, \mathbf{y}):=\sum_{q=1}^{Q}\left|x_{q}-y_{q}\right| .
$$

(This is the metric induced by the $\ell^{1}$ norm on $\left.\mathbb{R}^{Q}\right)$. Let $\Delta\left([0 \ldots M]^{Q}\right)$ be the set of all probability distributions on $[0 \ldots M]^{Q}$-that is, all functions $\nu:[0 \ldots M]^{Q} \longrightarrow[0,1]$ such that $\sum_{\mathbf{m} \in[0 \ldots M]^{Q}} \nu(\mathbf{m})=1$. For any $\nu \in \Delta\left([0 \ldots M]^{Q}\right)$ and all $q \in[1 \ldots Q]$, we define a nonincreasing function $\nu_{q}:[0 \ldots M+1] \longrightarrow[0,1]$ by

$$
\nu_{q}(n):=\sum\left\{\nu(\mathbf{m}) ; \mathbf{m}=\left(m_{1}, \ldots, m_{D}\right) \in[0 \ldots M]^{Q} \text { and } m_{q} \geq n\right\},
$$

for every $n \in[0 \ldots M]$, while $\nu_{q}(M+1):=0$. Let

$$
\Delta^{*}\left([0 \ldots M]^{Q}\right):=\left\{\nu \in \Delta\left([0 \ldots M]^{Q}\right) ; \nu_{q}(m) \neq \frac{1}{2}, \forall n \in[0 \ldots M] \text { and } \forall q \in[1 \ldots Q]\right\} .
$$

If $\nu \in \Delta^{*}\left([0 \ldots M]^{Q}\right)$, then the median of $\nu$ is the (unique) point $\mathbf{m}^{*} \in[0 \ldots M]^{Q}$ such that, for all $q \in[1 \ldots Q]$, we have $\nu_{q}\left(m_{q}^{*}\right)>\frac{1}{2}>\nu_{q}\left(m_{q}^{*}+1\right)$.

Lemma A.3 Define $\Phi:[0 \ldots M]^{Q} \longrightarrow\{0,1\}^{Q \times M}$ by equation (7) in $\S 3.4$.

(a) $\Phi$ is an isometry from the metric $d_{1}$ on $[0 \ldots M]^{Q}$ to the Hamming metric on $\{0,1\}^{Q \times M}$. That is: for all $\mathbf{x}, \mathbf{y} \in[0 \ldots M]^{Q}$, we have $d_{H}(\Phi(\mathbf{x}), \Phi(\mathbf{y}))=d_{1}(\mathbf{x}, \mathbf{y})$.

(b) Let $P \subseteq[0 \ldots M]^{Q}$ and let $X:=\Phi[P] \subset\{0,1\}^{Q \times M}$. Let $\mu \in \Delta^{*}(X)$. Define $\nu \in \Delta\left([0 \ldots M]^{Q}\right)$ by $\nu(\mathbf{m}):=\mu[\Phi(\mathbf{m})]$ for all $\mathbf{m} \in[0 \ldots M]^{Q}$. Then $\nu \in \Delta^{*}\left([0 \ldots M]^{Q}\right)$. If $\mathbf{m}^{*}$ is the median of $\nu$, then $\Phi\left(\mathbf{m}^{*}\right)=\operatorname{Maj}(\mu)$.

(c) Thus, if $S$ is the set of all points in $P$ of minimal $d_{1}$-distance from $\mathbf{m}^{*}$, then Slater $(X, \mu)=\Phi[S]$.

For any $r>0$, let $\diamond\left(\mathbf{m}^{*}, r\right):=\left\{\mathbf{n} \in \mathbb{Z}^{Q} ; d_{1}\left(\mathbf{n}, \mathbf{m}^{*}\right)=r\right\}$ be the 'sphere' of radius $r$ around the median $\mathbf{m}^{*}$ in the $d_{1}$ metric on $\mathbb{Z}^{Q}$ - this will be a polytope with $2^{Q}$ faces, each of which is a $Q$-dimensional simplex. (For example, if $Q=3$, then $\diamond\left(\mathbf{m}^{*}, r\right)$ is a regular octahedron). In Lemma A.3(c), we have $S=P \cap \diamond\left(\mathbf{m}^{*}, r\right)$ for some $r \in \mathbb{N}$. If $P=\triangle_{M}^{Q}$ is the simplex defined in eqn.(8), then typically this intersection will be between $P$ and one of the two simplicial faces of $\diamond\left(\mathbf{m}^{*}, r\right)$ which lies parallel to $P$ - see Figure 4 .

Proof of Lemma A.3. (a) Let $\mathbf{x}=\left(x_{1}, \ldots, x_{D}\right)$ and $\mathbf{y}=\left(y_{1}, \ldots, y_{D}\right)$ be elements of $[0 \ldots M]^{Q}$. Let $\widetilde{\mathbf{x}}:=\Phi(\mathbf{x})$ and $\widetilde{\mathbf{y}}:=\Phi(\mathbf{y})$ (elements of $\left.\{0,1\}^{M \times Q}\right)$. For all $q \in[1 \ldots Q]$, if $x_{q}<y_{q}$, then we have $\widetilde{x}_{(q, m)}=0 \neq 1=\widetilde{y}_{(q, m)}$ for all $m \in\left[x_{q}+1 \ldots y_{q}\right]$. If $y_{q}<x_{q}$, then we have $\widetilde{y}_{(q, m)}=0 \neq 1=\widetilde{x}_{(q, m)}$ for all $m \in\left[y_{q}+1 \ldots x_{q}\right]$. Either way, $\widetilde{x}_{(q, m)}=\widetilde{y}_{(q, m)}$ 
for all other $m \in[1 \ldots M]$, so that $\widetilde{\mathbf{x}}$ and $\widetilde{\mathbf{y}}$ differ in exactly $\left|x_{q}-y_{q}\right|$ of the coordinates $(q, 1), \ldots,(q, M)$. This holds for all $q \in[1 . . Q]$; hence $d_{H}(\widetilde{\mathbf{x}}, \widetilde{\mathbf{y}})=\left|x_{1}-y_{1}\right|+\cdots+\mid x_{D}-$ $y_{D} \mid=d_{1}(\mathbf{x}, \mathbf{y})$.

(b) Let $\mathbf{x}:=\operatorname{Maj}(\mu)$; we must show that $\mathbf{x}=\Phi\left(\mathbf{m}^{*}\right)$. For all $q \in[1 \ldots Q]$ and $n \in[1 \ldots M]$, combining the defining equations (1), (7), and (A3) yields $\mu_{(q, n)}(1)=\nu_{q}(n)$. Thus,

$$
\begin{aligned}
\left(x_{(q, n)}=1\right) & \Longleftrightarrow\left(\mu_{(q, n)}(1)>\frac{1}{2}\right) \Longleftrightarrow\left(\nu_{q}(n)>\frac{1}{2}\right) \\
& \Longleftrightarrow\left(m_{q}^{*} \geq n\right) \Longleftrightarrow\left(\Phi\left(\mathbf{m}^{*}\right)_{(q, n)}=1\right) .
\end{aligned}
$$

Thus, $x_{(q, n)}=\Phi\left(\mathbf{m}^{*}\right)_{(q, n)}$, for all $q \in[1 \ldots Q]$ and $n \in[1 \ldots M]$, as desired.

(c) This follows by combining (a) and (b).
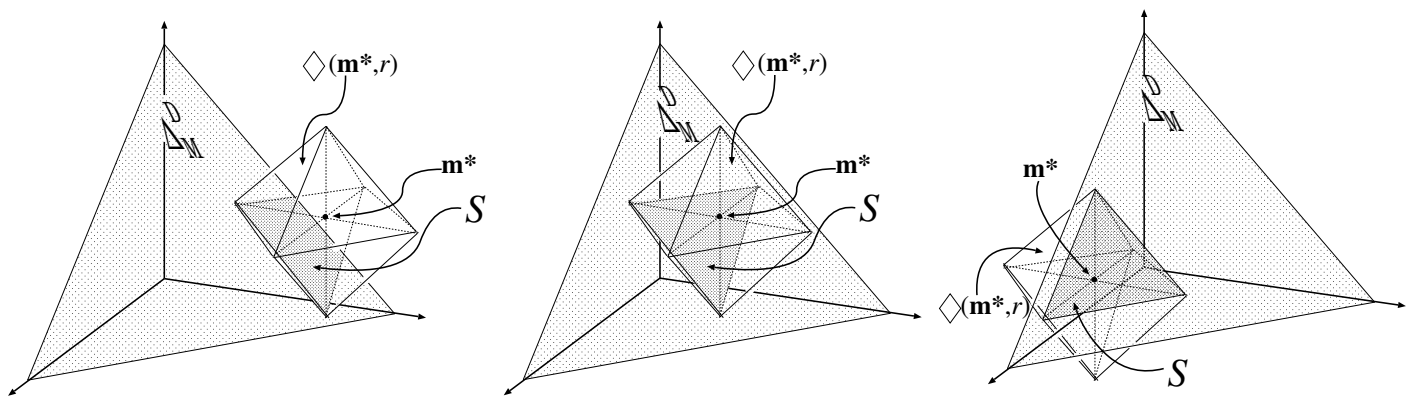

Figure 4: The Condorcet (=Slater) rule on the simplex. Here, $\operatorname{Maj}\left(X_{\vec{M}, Q}^{\Delta}, \mu\right)=\Phi\left[\mathbf{m}^{*}\right]$ and Cond $\left(X_{M, Q}^{\Delta}, \mu\right)=$ Slater $\left(X_{M, Q}^{\Delta}, \mu\right)=\Phi[S]$, where $S$ is the intersection of $\triangle_{M}^{Q}$ with another, parallel simplex. Figures (a) and (b) illustrate the case when $\mathbf{m}^{*}$ is above the simplex. (c) illustrates the case when $\mathbf{m}^{*}$ is below the simplex.

Proof of Proposition 3.9. Let $\mathbf{m}^{*} \in[0 \ldots M]^{Q}$ be the median point from Lemma A.3(b); thus, $\Phi\left(\mathbf{m}^{*}\right)=\operatorname{Maj}(\mu)$. Note that $0 \leq m_{q}^{*} \leq M$, for all $q \in[1 \ldots Q]$ (because every point in $X_{M, Q}^{\Delta}$ satisfies these constraints). There are three cases: either $\sum_{q=1}^{Q} m_{q}^{*}=M$, or $\sum_{q=1}^{Q} m_{q}^{*}>M$, or $\sum_{q=1}^{Q} m_{q}^{*}<M$

If $\sum_{q=1}^{Q} m_{q}^{*}=M$, then $\operatorname{Maj}(\mu) \in X_{M, Q}^{\Delta}$, in which case Cond $\left(X_{M, Q}^{\Delta}, \mu\right)=\operatorname{Maj}(\mu)$ by Lemma 1.5(a). Clearly, Slater $(X, \mu)=\operatorname{Maj}(\mu)$ also, so we're done.

If $\sum_{q=1}^{Q} m_{q}^{*}>M$, then $\mathbf{m}^{*}$ lies 'above' the simplex $\triangle_{M}^{Q}$ (as in Figures $\left.4(\mathrm{a}, \mathrm{b})\right)$. Let $O:=$ $\left\{\mathbf{n} \in \mathbb{Z}^{Q} ; n_{q} \leq m_{q}^{*}, \forall q \in[1 . . Q]\right\}$. (Heuristically, $O$ is the 'negative orthant' with its 
origin at $\mathbf{m}^{*}$.) Let $S:=O \cap \triangle_{M}^{Q}$; then it is easy to verify that Cond $\left(X_{M, Q}^{\Delta}, \mu\right)=\Phi[S]$, and satisfies the formula in Proposition 3.9(b). However, if $R:=D(\mu)=\sum_{q=1}^{Q} m_{q}^{*}-M$, then we have $S=\diamond\left(\mathbf{m}^{*}, R\right) \cap \triangle_{M}^{Q}$-in other words, $S$ is the set of points in $\triangle_{M}^{Q}$ minimizing the $d_{1}$-distance to $\mathbf{m}^{*}$; thus, Lemma A.3(c) says $\operatorname{Slater}\left(X_{M, Q}^{\Delta}, \mu\right)=\Phi[S]$. Thus, Slater $\left(X_{M, Q}^{\Delta}, \mu\right)=$ Cond $\left(X_{M, Q}^{\Delta}, \mu\right)$, as claimed.

If $\sum_{q=1}^{Q} m_{q}^{*}<M$, then the argument is similar, except that now $\mathbf{m}^{*}$ lies 'below' $\triangle_{M}^{Q}$ (as in Figures 4(c)), so we define $O$ to be the 'positive' orthant based at $\mathbf{m}^{*}$, which we intersect with $\triangle_{M}^{Q}$.

Proof of Proposition 3.10. (b) follows immediately from Proposition 3.9(b,c).

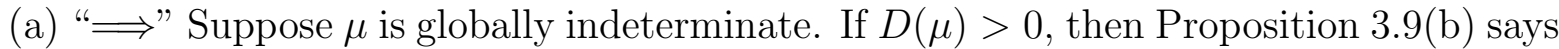
that $\mu$ can only be globally indeterminate if $D(\mu) \geq M$ and $m_{q}^{*}=M$ for all $q \in[1 \ldots Q]$. But if $m_{q}^{*}=M$ for some $q \in[1 \ldots Q]$, then $\mu[\mathbf{x}] \geq \frac{1}{2}$, where $\mathbf{x}$ is the allocation where all $M$ dollars go to claimant $q$; thus, $m_{p}^{*}=0$ for all other $p \in[1 \ldots Q] \backslash\{q\}$. Thus, it is impossible to have $m_{q}^{*}=M$ for all $q \in[1 \ldots Q]$.

So, suppose instead that $D(\mu)<0$. Then Proposition 3.9(c) says that $\mu$ can only be globally indeterminate if $D(\mu) \leq-M$ and $m_{q}^{*}=0$ for all $q \in[1 \ldots Q]$.

"£" Conversely, suppose $m_{q}^{*}=0$ for all $q \in[1 \ldots Q]$. Then $D(\mu)=\sum_{q=1}^{Q} m_{q}^{*}-M=$ $-M$. Thus, Proposition 3.9(c) implies that $\mu$ is globally indeterminate.

Proof of Corollary 3.11. Suppose $\mu \in \Delta^{*}\left(X_{M, Q}^{\Delta}\right)$ is such that every agent's allocation involves a positive quantity of more than half of the goods. Then for some $q \in[1 \ldots Q]$, more than half the agents support allocating at least one dollar to $q$, so $m_{q}^{*} \geq 1$. Thus, Proposition 3.10(a) implies that $\mu$ is not globally indeterminate.

Proof of Proposition 4.2. (a) was already proved in the paragraphs prior to Proposition 4.2 .

(b) Let $\mathbf{x} \in\{0,1\}^{K}$ be a graph that partitions the set $[1 \ldots N]$ into two cliques $A_{1}$ and $A_{2}$ and that contains exactly one additional edge connecting some $a \in A_{1}$ with some $b \in A_{2}$. Clearly, inc $(\mathbf{x})=1$ (remove the additional edge, to obtain an element of $X_{N}^{\text {eq }}$ with two equivalence classes). Since $X_{N}^{\mathrm{eq}}$ is McGarvey, there exists some $\mu \in \Delta\left(X_{N}^{\mathrm{eq}}\right)$ such that $\operatorname{Maj}(\mu)=\mathbf{x}$. Finally, the graph defined by $\mathbf{x}$ is connected, but not complete. Thus, Theorem 2.2(a) and Proposition 3.4(c) imply that $\mu$ is globally indeterminate.

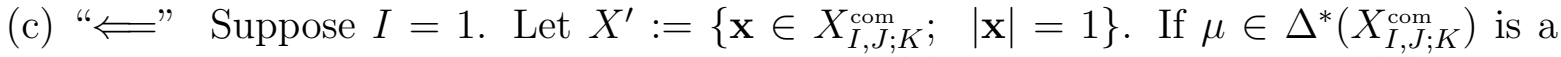
profile uniformly distributed over all $X^{\prime}$, then $\operatorname{Maj}(\mu)=\mathbf{0}$, so Proposition 3.5(b) implies 
that Cond $\left(X_{I, J ; K}^{\mathrm{com}}, \mu\right)=X^{\prime}$; thus, $\mu$ is globally indeterminate. But inc $(\mathbf{0})=1$ because $I=1$; thus, $X_{I, J ; K}^{\text {com }}$ is McKelvey.

The proof when $J=K-1$ is very similar.

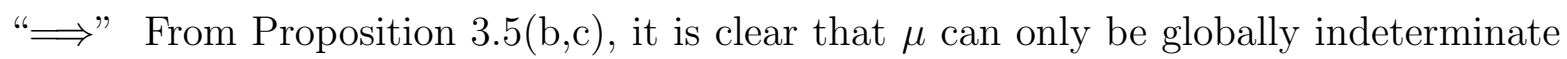
if $\operatorname{Maj}(\mu)=\mathbf{0}$ or $\operatorname{Maj}(\mu)=\mathbf{1}$. Thus, $X_{I, J ; K}^{\text {com }}$ can only be McKelvey if either $\operatorname{inc}(\mathbf{0})=1$ or $\operatorname{inc}(\mathbf{1})=1$. But inc $(\mathbf{0})=I$ and $\operatorname{inc}(\mathbf{1})=K-J$. Thus, $X_{I, J ; K}^{\text {com }}$ can only be McKelvey if either $I=1$ or $J=K-1$.

(d) Let $\mu \in \Delta^{*}\left(X_{M, Q}^{\Delta}\right)$. By Proposition 3.10(b) one has $\chi\left(X_{M, Q}^{\Delta}\right) \leq \frac{Q}{K}=\frac{1}{M}$. On the other hand, by part (a) of Proposition 3.10, for globally indeterminate profiles $\mu$, one obtains $\operatorname{inc}(\mu)=M$ and $\operatorname{ind}(\mu)=K$, hence $\chi\left(X_{M, Q}^{\Delta}\right) \geq \frac{1}{M}$. In particular, $X_{M, Q}^{\Delta}$ is never McKelvey.

\section{Towards the proof of Theorem 2.1}

The crux of path-dependence is that earlier precedents can override a majority in a later decision. An explanation of how this happens requires on a close analysis of the way that paths interact with critical fragments. Recall that $W(X, \mu)$ is the set of $X$-critical fragments activated by profile $\mu$. Let $\mathbf{w} \in W(X, \mu)$ and let $J:=\operatorname{supp}(\mathbf{w})$. If $\zeta$ is a path, and $k \in J$, then we say that $\zeta$ focuses $\mathbf{w}$ on $k$ if:

(F1) $\zeta$ covers every other element of $J$ before reaching $k$; and

(F2) for all $j \in J \backslash\{k\}$, we have $F_{j}^{\zeta}(\mu)=w_{j}=\operatorname{Maj}_{j}(\mu)$; hence

(F3) $F_{k}^{\zeta}(\mu)=\neg w_{k} \neq \operatorname{Maj}_{k}(\mu)$.

The following result is used to prove Theorem 2.1. It characterizes exactly when diachronic aggregation violates a majority in a particular coordinate.

Proposition A.4 Let $X \subseteq\{0,1\}^{K}$. Let $\mu \in \Delta^{*}(X)$, let $k \in[1 \ldots K]$, and let $\zeta$ be a path through $[1 \ldots K]$. Then:

$$
\left(F_{k}^{\zeta}(\mu) \neq \operatorname{Maj}_{k}(\mu)\right) \Longleftrightarrow(\text { There is some } \mathbf{w} \in W(X, \mu) \text { such that } \zeta \text { focuses } \mathbf{w} \text { on } k) \text {. }
$$

The "£" direction of Proposition A.4 follows immediately from (F3). The proof of

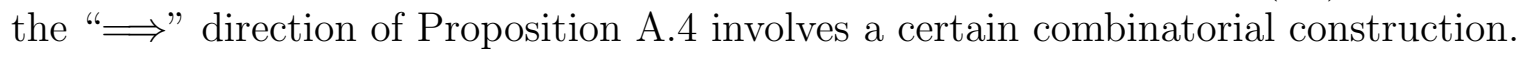

If $I, J \subseteq[1 \ldots K]$; then fragments $\mathbf{v} \in\{0,1\}^{I}$ and $\mathbf{w} \in\{0,1\}^{J}$ are compatible if $v_{k}=w_{k}$ for all $k \in I \cap J$ (hence, if $I \cap J=\emptyset$, then $\mathbf{v}$ and $\mathbf{w}$ are always compatible). In this case, we define $\mathbf{v} \uplus \mathbf{w} \in\{0,1\}^{I \cup J}$ by $(\mathbf{v} \uplus \mathbf{w})_{i}=v_{i}$ for all $i \in I$ and $(\mathbf{v} \uplus \mathbf{w})_{j}=w_{j}$ for all $j \in J$.

A forbidden tree of height 1 is a pair $T:=(\mathbf{w}, j)$, where $\mathbf{w}$ is a critical fragment (called the wood of $T$ ) and $j \in \operatorname{supp}(\mathbf{w})$. We say that $j$ is the root of $T$.

For any $h \geq 2$, we inductively define a forbidden tree of height $h$ to be a system $T:=$ $\left(\mathbf{w}, j ; T_{1}, \ldots, T_{N}\right)$, such that: 
(T1) $\mathbf{w}$ is a critical fragment, and $j \in \operatorname{supp}(\mathbf{w})$. (Here, $j$ is called the root of $T$, and $\mathbf{w}$ is the trunk of $T$.)

(T2) For all $n \in[1 \ldots N], T_{n}$ is a forbidden tree of height $h-1$ or less, whose root $j_{n}$ is an element of $\operatorname{supp}(\mathbf{w}) \backslash\{j\}$.

(T3) For all $n \in[1 \ldots N]$, if subtree $T_{n}$ has trunk $\mathbf{w}^{n}$, then $w_{j_{n}}^{n}=\neg w_{j_{n}}$.

(T4) For all $n \in[1 \ldots N]$, let $\widetilde{\mathbf{w}}^{n}$ be the wood of $T_{n}$, and let $J_{n}:=\operatorname{supp}\left(\widetilde{\mathbf{w}}^{n}\right) \backslash\left\{j_{n}\right\}$. Finally let $J_{0}:=J \backslash\left\{j_{1}, \ldots, j_{n}\right\}$. Then the subfamilies $\mathbf{w}_{J_{0}}, \widetilde{\mathbf{w}}_{J_{1}}^{1}, \widetilde{\mathbf{w}}_{J_{2}}^{2}, \ldots, \widetilde{\mathbf{w}}_{J_{N}}^{N}$ are all compatible. The wood of $T$ is the fragment $\widetilde{\mathbf{w}}:=\mathbf{w}_{J_{0}} \uplus \widetilde{\mathbf{w}}_{J_{1}}^{1} \uplus \widetilde{\mathbf{w}}_{J_{2}}^{2} \uplus \cdots \uplus \widetilde{\mathbf{w}}_{J_{N}}^{N}$.

Note: The wood of $T$ includes the root of $T$, but not the roots of its subtrees $T_{1}, \ldots, T_{N}$. In contrast, the support of $T$ is defined inductively:

$$
\operatorname{supp}(T) \quad:=\operatorname{supp}(\mathbf{w}) \cup \bigcup_{n=1}^{N} \operatorname{supp}\left(T_{n}\right) .
$$

Example A.5 Let $K=36$, and identify $[1 \ldots K]$ with a $6 \times 6$ grid as shown in Figure $5(\mathrm{~A})$. Let $J_{1}:=\{1,2,3\}$ and suppose $\mathbf{w}^{1}:=(0,0,0) \in\{0,1\}^{J_{1}}$ is critical [see Figure 5(B)]. Then $T_{1}:=\left(\mathbf{w}^{1}, 2\right)$ is a forbidden tree of height 1 [see Figure $\left.5(\mathrm{C})\right]$. Let $J_{2}:=\{2,8,14,20,26,32\}$ and suppose $\mathbf{w}^{2}:=(1,1,1,1,1,1) \in\{0,1\}^{J_{2}}$ is critical [see Figure 5(D)]. Then $T_{2}:=$ $\left(\mathbf{w}^{2}, 26 ; T_{1}\right)$ is a forbidden tree of height 2 [see Figure $\left.5(\mathrm{E})\right]$. Let $J_{3}:=\{25,26,27,28,29\}$ and suppose $\mathbf{w}^{3}:=(0,0,0,0,0) \in\{0,1\}^{J_{3}}$ is critical [see Figure $\left.5(\mathrm{~F})\right]$. Then $T_{3}:=\left(\mathbf{w}^{3}, 28 ; T_{2}\right)$ is a forbidden tree of height 3 [see Figure $5(\mathrm{G})]$. Let $J_{4}:=\{14,15,16,17,18\}$ and suppose $\mathbf{w}^{4}:=(1,0,0,0,0) \in\{0,1\}^{J_{4}}$ is critical [see Figure $\left.5(\mathrm{H})\right]$. Then $T_{4}:=\left(\mathbf{w}^{4}, 16\right)$ is a forbidden tree of height 1 [see Figure 5(I)]. Finally, let $J_{5}:=\{16,22,28,34\}$ and suppose $\mathbf{w}^{5}:=$ $(1,1,1,1) \in\{0,1\}^{J_{5}}$ is critical [see Figure $5(\mathrm{~J})$ ]. Then $T_{5}:=\left(\mathbf{w}^{5}, 34 ; T_{3}, T_{4}\right)$ is a forbidden tree of height 4, shown in Figure 5(K). A 'schematic' of $T_{5}$ is shown in Figure 5(L). Observe that two 'branches' of $T_{5}$ overlap in coordinate 14 , but they are compatible because $w_{14}^{2}=1=w_{14}^{4}$; this is the significance of condition (T4). The support of $T_{5}$ is all entries in Figure $5(\mathrm{~K})$ containing ' 0 ', ' 1 ', or '*'. The wood of $T_{5}$ is all entries in Figure $5(\mathrm{~K})$ containing a ' 0 ' or a ' 1 ' (but not $\mathrm{a}^{(*)}$ ).

For any $\mu \in \Delta^{*}(X)$, we say that $\mu$ activates the tree $T$ if $\mu$ activates the wood of $T$.

Lemma A.6 Let $X \subseteq\{0,1\}^{K}$.

(a) Let $\widetilde{\mathbf{w}}$ be the wood of a forbidden tree. Then $\widetilde{\mathbf{w}}$ is itself a forbidden fragment for $X$.

(b) Let $\mu \in \Delta^{*}(X)$, let $k \in[1 \ldots K]$, and let $\zeta$ be a path such that $F_{k}^{\zeta}(\mu) \neq \operatorname{Maj}_{k}(\mu)$. Then $k$ is the root of a forbidden tree $T$ activated by $\mu$, such that:

(b1) $\zeta$ covers every other element of $\operatorname{supp}(T)$ before reaching $k$; and 


\begin{tabular}{|l|l|l|l|l|l|}
\hline 1 & 2 & 3 & 4 & 5 & 6 \\
\hline 7 & 8 & 9 & 10 & 11 & 12 \\
\hline 13 & 14 & 15 & 16 & 17 & 18 \\
\hline 19 & 20 & 21 & 22 & 23 & 24 \\
\hline 25 & 26 & 27 & 28 & 29 & 30 \\
\hline 31 & 32 & 33 & 34 & 35 & 36 \\
\hline$(A)$ & $K$
\end{tabular}

\begin{tabular}{|l|l|l|l|l|l|}
\hline 0 & $*$ & 0 & & & \\
\hline & 1 & & & & \\
\hline & 1 & & & & \\
\hline & 1 & & & & \\
\hline & 1 & & & & \\
\hline & 1 & & & & \\
(E) & \multicolumn{4}{|c|}{$T_{2}:=\left(\mathbf{w}^{2}, 26 ; T_{1}\right)$}
\end{tabular}

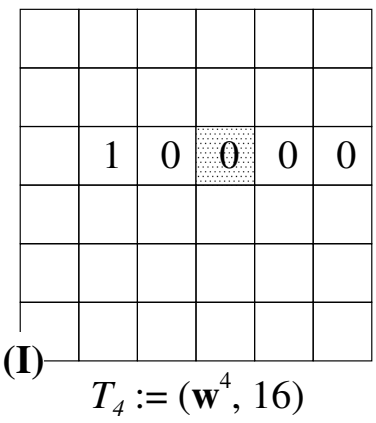

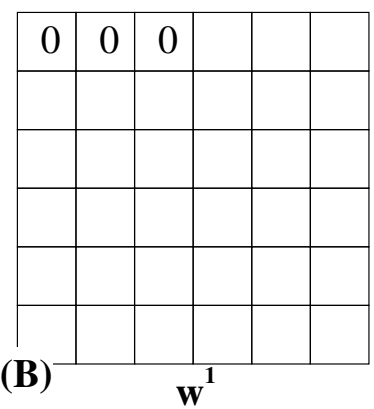
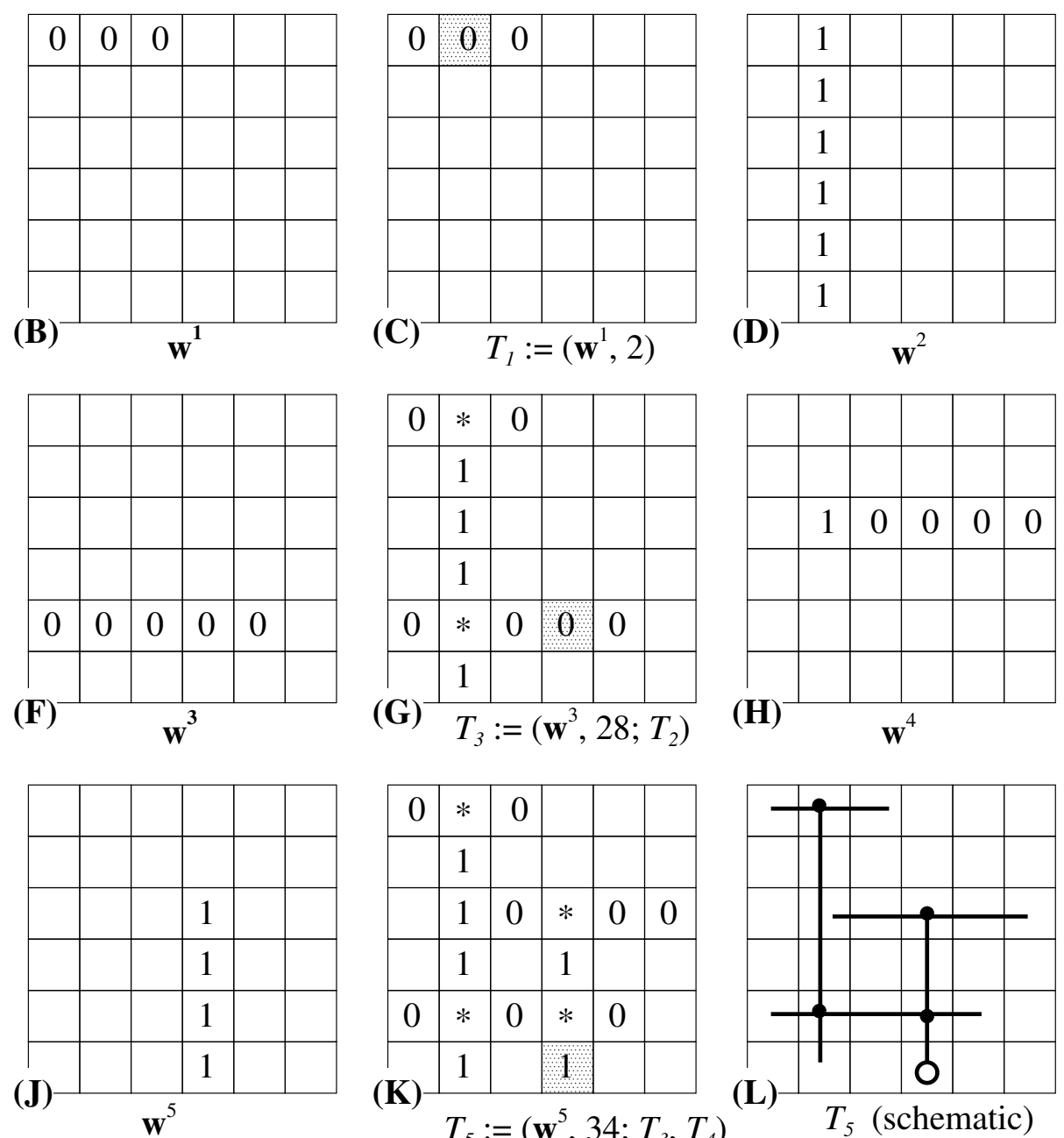

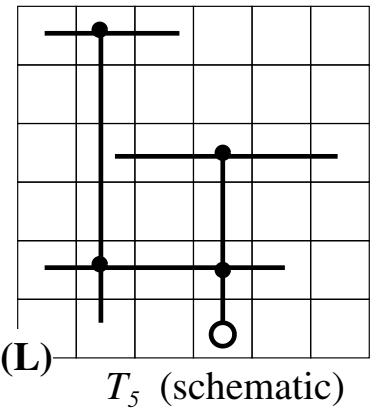

Figure 5: Construction of forbidden trees. Boxes labelled ' 0 ' or ' 1 ' are part of the wood of the tree. Boxes labelled '*' are in the support of the tree, but not its wood. The shaded boxes are the roots of the trees. See Example A.5 for explanation.

(b2) if $\widetilde{\mathbf{w}}$ is the wood of $T$, then for all $j \in \operatorname{supp}(\widetilde{\mathbf{w}}) \backslash\{k\}$ we have $F_{j}^{\zeta}(\mu)=\operatorname{Maj}_{j}(\mu)=$ $\widetilde{w}_{j}$.

Proof: (a) (by induction on height) If $T$ is a tree of height 1 , then $T:=(\mathbf{w}, j)$, and its wood is the forbidden fragment $\mathbf{w}$ by definition.

Now let $h \geq 2$ and inductively suppose the claim is true for all trees of height less than $h$. Let $T:=\left(\mathbf{w}, j, T_{1}, \ldots, T_{N}\right)$ be a forbidden tree of height $h$, with wood $\widetilde{\mathbf{w}}$ and let $\widetilde{J}=\operatorname{supp}(\widetilde{\mathbf{w}})$. Let $\mathbf{x} \in X$, and suppose (by contradiction) that $\mathbf{x}_{\widetilde{J}}=\widetilde{\mathbf{w}}$. Let $J:=\operatorname{supp}(\mathbf{w})$. For all $n \in[1 \ldots N]$, let forbidden tree $T_{n}$ have root $j_{n} \in J$.

Claim 1: (a) For all $n \in[1 \ldots N]$, we have $x_{j_{n}}=w_{j_{n}}$.

(b) Also, $\mathbf{x}_{J \backslash\left\{j_{1}, \ldots, j_{N}\right\}}=\mathbf{w}_{J \backslash\left\{j_{1}, \ldots, j_{N}\right\}}$.

Proof: (a) Fix $n \in[1 \ldots N]$. Let $T_{n}$ have $\operatorname{wood} \mathbf{w}^{n}$ and let $J_{n}:=\operatorname{supp}\left(\mathbf{w}^{n}\right)$; then 
$\widetilde{\mathbf{w}}_{J_{n} \cap \widetilde{J}}=\mathbf{w}_{J_{n} \cap \widetilde{J}}^{n}$ by definition of 'wood' in condition (T4). Thus, $\mathbf{x}_{J_{n} \cap \widetilde{J}}=\mathbf{w}_{J_{n} \cap \widetilde{J}}^{n}$ ' because $\mathbf{x}_{\widetilde{J}}=\widetilde{\mathbf{w}}$ by hypothesis. But $J_{n} \cap \widetilde{J}=J_{n} \backslash\left\{j_{n}\right\}$, so we have $\mathbf{x}_{J_{n} \backslash\left\{j_{n}\right\}}=\mathbf{w}_{J_{n} \backslash\left\{j_{n}\right\}}^{n}$. We must then have $x_{j_{n}}=\neg w_{j_{n}}^{n}$, because $\mathbf{w}^{n}$ is a forbidden fragment (by induction hypothesis). Thus, $x_{j_{n}}=w_{j_{n}}$, because $w_{j_{n}}=\neg w_{j_{n}}^{n}$ by (T3).

(b) $J \backslash\left\{j_{1}, \ldots, j_{N}\right\}=\widetilde{J} \cap J$ and $\widetilde{\mathbf{w}}_{\widetilde{J} \cap J}=\mathbf{w}_{\widetilde{J} \cap J}$ (by definition of 'wood' in (T4)); thus $\mathbf{x}_{J \backslash\left\{j_{1}, \ldots, j_{N}\right\}}=\mathbf{w}_{J \backslash\left\{j_{1}, \ldots, j_{N}\right\}}$ (because $\mathbf{x}_{\widetilde{J}}=\widetilde{\mathbf{w}}$ by hypothesis).

$\diamond$ Claim 1

Claim 1 implies that $\mathbf{x}_{J}=\mathbf{w}$. But $\mathbf{w}$ is a forbidden fragment (by (T1)). Contradiction. Thus, for all $\mathbf{x} \in X$ we must have $\mathbf{x}_{\widetilde{J}} \neq \widetilde{\mathbf{w}}$; hence $\widetilde{\mathbf{w}}$ is a forbidden fragment, as desired.

(b) Suppose $k=\gamma(t)$ for some $t \in[1 \ldots K]$. If $F_{k}^{\zeta}(\mu) \neq \operatorname{Maj}_{k}(\mu)$, then we must have $t \geq 2$. We will prove the claim by induction on $t$.

Base case. If $t=2$, then let $J:=\{\zeta(1), \zeta(2)\}$ and $\mathbf{w}:=\operatorname{Maj}_{J}(\mu)$; then $\mathbf{w}$ must be a critical fragment, so $(\mathbf{w}, k)$ is a forbidden tree of height 1 .

Induction. If $t \geq 3$, and $F_{k}^{\zeta}(\mu) \neq \operatorname{Maj}_{k}(\mu)$, then there must exist some subset $J \subseteq$ $\{\zeta(1), \ldots, \zeta(t)\}$ including $k=\zeta(t)$ such that, if we define $w_{k}:=\operatorname{Maj}_{k}(\mu)$, and define $w_{j}:=F_{j}^{\zeta}(\mu)$ for all $j \in J \backslash\{k\}$, then $\mathbf{w} \in\{0,1\}^{J}$ is a forbidden fragment for $X$. By choosing $J$ to be a minimal subset with this property, we can assume $\mathbf{w}$ is critical.

Let $\left\{j_{1}, \ldots, j_{N}\right\}$ be the set of all elements of $J \backslash\{k\}$ such that $\operatorname{Maj}_{j_{n}}(\mu) \neq w_{j_{n}}$. By induction hypothesis, each $j_{n}$ is the root of a $\mu$-activated forbidden tree $T_{n}$ such that

$\left(\mathrm{b1}^{\prime}\right) \zeta$ covers every other element of $\operatorname{supp}\left(T_{n}\right)$ before reaching $j_{n}$; and

(b2') if $\mathbf{w}^{n}$ is the wood of $T_{n}$ and $J_{n}:=\operatorname{supp}\left(\mathbf{w}^{n}\right)$, then for all $j \in J_{n} \backslash\left\{j_{n}\right\}$, we have $F_{j}^{\zeta}(\mu)=\operatorname{Maj}_{j}(\mu)=w_{j}^{n}$.

Let $T:=\left(\mathbf{w}, k ; T_{1}, \ldots, T_{N}\right)$; we claim $T$ is a forbidden tree. (T1) is true because $\mathbf{w}$ is a critical fragment by construction. (T2) is true by definition of $T_{1}, \ldots, T_{n}$. To see (T3), note for all $n \in[1 \ldots N]$ that $\mu$ activates $\mathbf{w}^{n}$ by hypothesis, so $w_{j_{n}}^{n}=\operatorname{Maj}_{j_{n}}(\mu)$, whereas $w_{j_{n}} \neq \operatorname{Maj}_{j_{n}}(\mu)$ by definition of $\left\{j_{1}, \ldots, j_{N}\right\}$; thus $w_{j_{n}}^{n}=\neg w_{j_{n}}$. To see (T4), observe that the woods of $T_{1}, \ldots, T_{N}$ are all compatible because they are all subfamilies of $F^{\zeta}(\mu)$, by condition (b2'). Finally, properties (b1) and (b2) follow immediately from the definitions of $J$ and $T$, and properties $\left(\mathrm{b} 1^{\prime}\right)$ and $\left(\mathrm{b} 2^{\prime}\right)$.

Proof of Proposition A.4 “ $\Longrightarrow$ ". Let $\mu, k$, and $\zeta$ by as in the statement of the Proposition. Lemma A.6(b) says that $k$ is the root of a $\mu$-activated forbidden tree $T$ satisfying (b1) and (b2). Let $\widetilde{\mathbf{w}}$ be the wood of $T$; then Lemma A.6(a) says that $\widetilde{\mathbf{w}}$ is itself a forbidden fragment. Let $\widetilde{J}:=\operatorname{supp}(\widetilde{\mathbf{w}})$; then $k \in \widetilde{J}$, and $\widetilde{\mathbf{w}}_{\widetilde{J} \backslash\{k\}}$ is not forbidden, by (b2). Thus, any forbidden subfragment of $\widetilde{\mathbf{w}}$ must contain coordinate $k$. Since $\widetilde{J}$ is finite, there exists some $J \subseteq \widetilde{J}$ (with $k \in J$ ) such that $\mathbf{w}:=\widetilde{\mathbf{w}}_{J}$ is a minimal forbidden subfragment -i.e. a critical fragment. At this point, (F1) follows from (b1), (F2) follows from (b2), and (F3) is true because $F_{k}^{\zeta}(\mu) \neq \operatorname{Maj}_{k}(\mu)$ by hypothesis. 
Proof of Theorem 2.1. We first establish two claims.

Claim 1: $\operatorname{Indet}(\mu)=\left\{k \in[1 \ldots K]\right.$; there exists a path $\gamma$ such that $\left.F_{k}^{\gamma}(\mu) \neq \operatorname{Maj}_{k}(\mu)\right\}$.

Proof: "ᄃ" Proposition 1.8(b) says that $k \in \operatorname{Indet}(\mu)$ if and only if there are paths $\gamma$ and $\zeta$ such that $F_{k}^{\gamma}(\mu) \neq F_{k}^{\zeta}(\mu)$. Either $F_{k}^{\gamma}(\mu)=\operatorname{Maj}_{k}(\mu)$ or $F_{k}^{\zeta}(\mu)=\operatorname{Maj}_{k}(\mu)$. If $F_{k}^{\zeta}(\mu)=\operatorname{Maj}_{k}(\mu)$, then $F_{k}^{\gamma}(\mu) \neq \operatorname{Maj}_{k}(\mu)$.

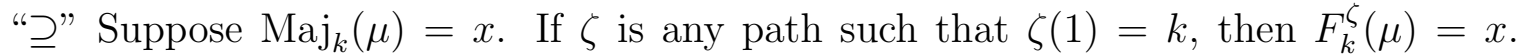
Thus, if $\gamma$ is some path such that $F_{k}^{\gamma}(\mu) \neq \operatorname{Maj}_{k}(\mu)$, then $F_{k}^{\gamma}(\mu) \neq F_{k}^{\zeta}(\mu)$; hence $k \in \operatorname{Indet}(\mu)$.

$\diamond$ Claim 1

Claim 2: $\quad$ For any $\mathbf{w} \in W(X, \mu)$ and $k \in \operatorname{supp}(\mathbf{w})$, there is a path focusing $\mathbf{w}$ on $k$.

Proof: $\operatorname{Suppose} \operatorname{supp}(\mathbf{w})=J:=\left\{j_{1}, j_{2}, \ldots, j_{N}\right\}$, where $j_{N}=k$. Let $\gamma$ be a path such that $\gamma(n)=j_{n}$ for all $n \in[1 \ldots N]$, after which $\gamma$ traverses the rest of $[1 \ldots K]$ in some order (this gives (F1)). For all $j \in J$ we have $\operatorname{Maj}_{j}(\mu)=w_{j}$ (because w is activated by $\mu$, by hypothesis). Thus, we have $F_{j}^{\gamma}(\mu)=w_{j}$ for all $j \in J \backslash\{k\}$ (because $\mathbf{w}_{J \backslash\{k\}}$ is not forbidden, because $\mathbf{w}$ is critical); this yields (F2). But then $F_{k}^{\gamma}(\mu) \neq w_{k}$, because $\mathbf{w}$ is forbidden in $X$; this yields (F3).

$\diamond$ Claim 2

Now let $B:=\bigcup_{\mathbf{w} \in W(X, \mu)} \operatorname{supp}(\mathbf{w})$. We must show that $\operatorname{Indet}(\mu)=B$.

We have Indet $(\mu) \subseteq B$ by Claim 1 and Proposition A.4 “ $\Longrightarrow$ ".

To see that $\operatorname{Indet}(\mu) \supseteq B$, let $\mathbf{w} \in W(X, \mu)$, and let $k \in \operatorname{supp}(\mathbf{w})$. Claim 2 says there is a path $\gamma$ which focuses $\mathbf{w}$ on $k$. Then Proposition A.4 "£" implies that $F_{k}^{\gamma}(\mu) \neq$ $\operatorname{Maj}_{k}(\mu)$; hence Claim 1 says $k \in \operatorname{Indet}(\mu)$.

\section{References}

Baldiga, K., 2012. Representative democracy and the implementation of majority-preferred alternatives. (preprint).

Barthélémy, J.-P., 1989. Social welfare and aggregation procedures: combinatorial and algorithmic aspects. In: Applications of combinatorics and graph theory to the biological and social sciences. Vol. 17 of IMA Vol. Math. Appl. Springer, New York, pp. 39-73.

Barthélémy, J.-P., Janowitz, M. F., 1991. A formal theory of consensus. SIAM J. Discrete Math. 4 (3), $305-322$.

Barthélémy, J.-P., Monjardet, B., 1981. The median procedure in cluster analysis and social choice theory. Math. Social Sci. 1 (3), 235-267.

Barthélémy, J.-P., Monjardet, B., 1988. The median procedure in data analysis: new results and open problems. In: Classification and related methods of data analysis (Aachen, 1987). North-Holland, Amsterdam, pp. 309-316.

Dasgupta, P., Maskin, E., 2004. The fairest vote of all. Scientific American March, 92-97. 
Dietrich, F., List, C., 2007. Judgement aggregation by quota rules: Majority voting generalized. Journal of Theoretical Politics 19 (4), 391-424.

Dietrich, F., List, C., 2010. Majority voting on restricted domains. Journal of Economic Theory 145 (2), $512-543$.

Dimitrov, D., Marchant, T., Mishra, D., 2012. Separability and aggregation of equivalence relations. Economic Theory (to appear).

Dokow, E., Holzman, R., 2010. Aggregation of binary evaluations. J.Econ.Theory 145, 495-511.

Duggan, J., 2007. A systematic approach to the construction of non-empty choice sets. Social Choice and Welfare 28, 491-506.

Fishburn, P., Rubinstein, A., 1986. Aggregation of equivalence relations. Journal of classification 3, 61-65.

Good, I. J., 1971. A note on condorcet sets. Public Choice 10, 97-101.

Guilbaud, T., 1952. Les theories de l'interet general et le probleme logique de l'aggregation. Econ. Appl. 5 (4), 501-584, complete English translation in: Electronic J. History Probab. Statist. 4 (2008).

Kemeny, J. G., 1959. Math without numbers. Daedalus 88, 571-591.

Kornhauser, L., Sager, L., 1986. Unpacking the court. Yale Law Journal 96, 82-117.

Lang, S., Pigozzi, G., Slavkovik, M., van der Torre, L., 2011. Judgement aggregation rules based on minimization. In: Proceedings of the 13th Conference on Theoretical Aspects of Rationality and Knowledge. ACM, New York, NY, pp. 238-246.

Laslier, J.-F., 1997. Tournament Solutions and Majority Voting. Springer, Berlin.

Lindner, T., Nehring, K., Puppe, C., 2010. Allocating public goods via the midpoint rule. (preprint).

List, C., 2004. A model of path-dependence in decisions over multiple propositions. American Political Science Review 98 (3), 495-513.

List, C., Pettit, P., 2002. Aggregating sets of judgements: An impossibility result. Economics and Philosophy $18,89-110$.

List, C., Puppe, C., 2009. Judgement aggregation: a survey. In: Anand, P., Pattanaik, P., Puppe, C. (Eds.), Oxford handbook of rational and social choice. Oxford University Press, Oxford, UK., Ch. 19, pp. $457-481$.

May, K., 1952. A set of independent, necessary, and sufficient conditions for simple majority decision. Econometrica 20, 680-684.

McGarvey, D. C., 1953. A theorem on the construction of voting paradoxes. Econometrica 21, 608-610.

McKelvey, R., 1979. General conditions for global intransitivities in formal voting models. Econometrica 47 (5), 1085-1112.

McLean, I., 1990. The Borda and Condorcet principles: Three medieval applications. Social Choice and Welfare 7, 99-108.

Miller, A., 2009. A model of community standards. (preprint). 
Miller, N. R., 1977. Graph-theoretical approaches to the theory of voting. American Journal of Political Science 21, 769-803.

Moulin, H., 1988. Axioms of cooperative decision making. Cambridge University Press, Cambridge, UK.

Nehring, K., Pivato, M., 2011. Incoherent majorities: the McGarvey problem in judgement aggregation. Discrete Appl. Math. 159 (15), 1488-1507.

Nehring, K., Pivato, M., 2012. Majority rule without a majority. (preprint).

Nehring, K., Pivato, M., Puppe, C., 2013. Unanimity overruled: Majority voting and the burden of history. (preprint).

Nehring, K., Puppe, C., 2007. The structure of strategy-proof social choice I: General characterization and possibility results on median spaces. J.Econ.Theory 135, 269-305.

Nehring, K., Puppe, C., 2010. Abstract arrowian aggregation. J.Econ.Theory 145, 467-494.

Pivato, M., 2009. Geometric models of consistent judgement aggregation. Social Choice and Welfare 33 (4), $559-574$.

Risse, M., 2004. Arguing for majority rule. The Journal of Political Philosophy 12, 41-64.

Sen, A. K., 1970. Collective Choice and Social Welfare. Holden Day, San Francisco.

Slater, P., 1961. Inconsistencies in a schedule of paired comparisons. Biometrica 48, 303-312.

Waldron, J., 1999. The Dignity of Legislation. Cambridge University Press, Cambridge.

Young, H. P., Levenglick, A., 1978. A consistent extension of Condorcet's election principle. SIAM J. Appl. Math. 35 (2), 285-300. 


\section{Working Paper Series in Economics}

recent issues

No. 51 Klaus Nehring, Marcus Pivato, Clemens Puppe: The Condorcet set: majority voting over interconnected propositions, December 2013

No. 50 Klaus Nehring, Marcus Pivato, Clemens Puppe: Unanimity overruled: majority voting and the burden of history, December 2013

No. 49 Andranik S. Tangian: Decision making in politics and economics: 5. 2013 election to German Bundestag and direct democracy, December 2013

No. 48 Marten Hillebrand, Tomoo Kikuchi, Masaya Sakuragawa: Bubbles and crowding-in of capital via a savings glut, November 2013

No. 47 Dominik Rothenhäusler, Nikolaus Schweizer, Nora Szech: Institutions, shared guilt, and moral transgression, October 2013

No. 46 Marten Hillebrand: Uniqueness of Markov equilibrium in stochastic OLG models with nonclassical production, November 2012

No. 45 Philipp Schuster and Marliese Uhrig-Homburg: The term structure of bond market liquidity conditional on the economic environment: an analysis of government guaranteed bonds, November 2012

No. 44 Young Shin Kim, Rosella Giacometti, Svetlozar T. Rachev, Frank J. Fabozzi, Domenico Mignacca: Measuring financial risk and portfolio optimization with a non-Gaussian multivariate model, August 2012

No. 43 Zuodong Lin, Svetlozar T. Rachev, Young Shin Kim, Frank J. Fabozzi: Option pricing with regime switching tempered stable processes, August 2012

No. 42 Siegfried K. Berninghaus, Werner Güth, Stephan Schosser: Backward induction or forward reasoning? An experiment of stochastic alternating offer bargaining, July 2012

No. 41 Siegfried Berninghaus, Werner Güth, King King Li: Approximate truth of perfectness - an experimental test, June 2012 